\title{
Gelation/crystallization mechanisms of UHMWPE solutions and structures of ultradrawn gel films
}

\author{
Xiaomei Shi ${ }^{1}$, Yuezhen Bin $^{1}$, Daishui Hou ${ }^{1}$, Yongfeng Men ${ }^{2}$ and Masaru Matsuo ${ }^{1}$ \\ The gelation mechanisms of ultra-high-molecular-weight polyethylene (UHMWPE) chains differed significantly in decalin and \\ paraffin, although these are typical solvents used to gel spin UHMWPE fibers with high strengths and moduli. In both solvents, \\ gelation was attributed to liquid-liquid phase separation driven by concentration fluctuations during the initial stage. The \\ gelation speeds and temperatures differed significantly in the two solvents. In decalin, solvent flowed from the gels, syneresis \\ occurred during crystallization and dry gel films were prepared. In contrast, in paraffin, many nucleating points appeared \\ immediately, and no syneresis occurred even after quenching to room temperature. To prepare dry gel films, paraffin was blotted \\ with filter paper, and paraffin residues were removed in hexane. However, the small-angle X-ray scattering spectra from both \\ films were similar, indicating crystalline lamellae and ultradrawing.
}

Polymer Journal (2014) 46, 21-35; doi:10.1038/pj.2013.66; published online 31 July 2013

Keywords: gel syneresis; gelation speed and temperature; high-strength, high-modulus UHMWPE; liquid-liquid phase separation; theoretical results for SAXS

\section{INTRODUCTION}

Gel deformation is an excellent method for the production of highmodulus, high-strength polyethylene (PE) films. Dry gel films and single crystal mats were produced by crystallizing ultra-high-molecular-weight polyethylene (UHMWPE) with a molecular weight $>1 \times 10^{6}$ from decalin. ${ }^{1-13}$ This gel method was based on the assumption that the maximum achievable draw ratio, over 200, depends primarily on the solution concentration of the polymer. This dependence was attributed to a lower number of entanglements per molecule in solutions than in melts for cast/spun polymers. ${ }^{2,9,12,13}$ If this is the case, then solvent types as well as concentrations are relevant. The optimum level of entanglements within dry gel films is determined by the macromolecular size in solution. Both $p$-xylene and decalin have been used widely as solvents for polyethylene to produce single crystal mats of UHMWPE. ${ }^{14-16}$

In addition to decalin and $p$-xylene, paraffin has been used as a solvent to prepare high-strength, high-modulus UHMWPE fibers by gel spinning in commercial production. However, there are fewer detailed investigations of the gelation mechanism and dry gel film formation from paraffin than from decalin and $p$-xylene. Paraffin has many advantages, as UHMWPE solutions with concentrations $>30 \%$ can be extruded and immersed in hexane to remove the paraffin. Then, the wet gel can be stretched stepwise in an oven at a controlled temperature. ${ }^{17}$ Solvent recovery is easy with this method.
The factors that dominate gel formation and determine the characteristics of physical gels have not been determined. There are two primary views regarding physical gel formation. One approach concentrates on gel structures and equilibrium dynamics of linear polymers. ${ }^{18-26}$

The second approach focuses on the time dependence of gelation at various temperatures and applies light scattering, X-rays and neutron scattering to measure dynamic characteristics. ${ }^{27-33}$ The results indicate that when crystalline polymer solutions are quenched, liquid-liquid phase separation associated with quasi-spinodal decomposition occurs in the polymer-rich phase. The phase separation of $\mathrm{UHMWPE}^{32}$ and ultra-high-molecular-weight polypropylene ${ }^{30}$ during gelation was investigated relative to the morphology and mechanical properties of dry gel films. Dense network structures smoothly transmit internal stress in the stretching direction, which ensures ultradrawing by transformations from folded to extended crystals.

This paper addresses the second approach. Paraffin and decalin are used to identify different gelation/crystallization mechanisms of UHMWPE chains and lamellar formation in the two types of dry gel films.

As in previous work ${ }^{34,35}$ for gels prepared with decalin, polymerrich regions in two types of gels were analyzed using a threedimensional statistical theory, ${ }^{35}$ and the formation of a rod-like gel $^{34}$ was studied by polarized small-angle light scattering (SALS).

\footnotetext{
${ }^{1}$ Department of Polymer Science and Materials, School of Chemical Engineering, Dalian University of Technology, Dalian, People's Republic of China and 2State Key Laboratory of Polymer Physics and Chemistry, Changchun Institute of Applied Chemistry, Chinese Academy of Sciences, Graduate School of Chinese Academy of Sciences, Changchun, People's Republic of China

Correspondence: Professor M Matsuo, Department of Polymer Material Science, School of Chemical Engineering, Dalian University of Technology, No. 2 Linggong Road, Ganjingzi District, Dalian City, Liaoning Province 116024, China.

E-mail: mm-matsuo@live.jp

Received 22 April 2013; revised 20 May 2013; accepted 1 June 2013; published online 31 July 2013
} 
In decalin and paraffin, the formation of gel structures by liquidliquid phase separation differed, but the crystalline lamellae in the dry gel films were very similar according to small-angle X-ray scattering (SAXS), and the dry gel films could be stretched more than 150 times. These unusual phenomena must be elucidated.

In a prior study, gelation mechanisms in decalin were analyzed using SALS patterns. ${ }^{34,35}$ In that research, gelation mechanisms in decalin and paraffin were analyzed with wide-angle X-ray diffraction (WAXD) and SALS. In addition, a helium-neon gas laser was used to study the time dependence of crystallization in polymer-rich phases by the phase separation of the solutions. Furthermore, lamellar structures in the two types of dry gel films, ultradrawn more than 150 times, ${ }^{2,9}$ were analyzed by SAXS, ${ }^{36,37}$ particularly with respect to the excess electron density between the crystal and the amorphous regions.

\section{EXPERIMENTAL PROCEDURE}

\section{Materials and sample preparation}

UHMWPE with an average viscosity molecular weight of $3 \times 10^{6}$ and $\bar{M}_{w} / \bar{M}_{n}$ ca. 4.2 (measured by Gel Permeation Chromatography) was provided by Ticona (Wilmington, NC, USA). The UHMWPE was purified by Soxhlet extraction in ethanol for $12 \mathrm{~h}$. The decalin and paraffin were normal solvents used in industrial applications. The paraffin solvent had a broad molecular dispersion (carbon numbers $\mathrm{C}_{16}-\mathrm{C}_{31}$ ). In the present work, the concentration of UHMWPE was $1 \mathrm{~g}$ per $100 \mathrm{ml}$ with respect to the solvent. In the preliminary experiments, $1 \mathrm{~g}$ per $100 \mathrm{ml}$ was the best concentration for the preparation of UHMWPE/filler composites because the high viscosity provided a shear stress that ensured the uniform dispersion of particles. This finding was demonstrated for UHMWPE/carbon black, ${ }^{38}$ UHMWPE/carbon fibers, ${ }^{39}$ UHMWPE/ multiwall carbon nanotubes ${ }^{40,41}$ and UHMWPE/hydroxyapatite. ${ }^{42}$ The previous investigations of gelation mechanism of UHMWPE were performed in solutions with concentrations $<0.65 \mathrm{~g}$ per $100 \mathrm{ml},{ }^{12}$ as higher viscosity solutions $>1 \mathrm{~g}$ per $100 \mathrm{ml}$ could not be poured into $1 \mathrm{~mm}$ quartz cells for WAXD measurements. Accordingly, quartz cells with $2.5 \mathrm{~mm}$ diameters were used in the present studies of phase separation of UHMWPE chains in decalin and paraffin.

The mixture was heated to $145^{\circ} \mathrm{C}$ and stirred for $30 \mathrm{~min}$. Solutions were poured into test tubes and put in a silicon bath controlled in the range from 70 to $120^{\circ} \mathrm{C}$.

To determine the gelation time with respect to temperature, test tubes containing UHMWPE solutions were tilted in a water bath maintained at a constant temperature. The gelation time was determined when the meniscus deformed but the specimen did not flow under its own weight. ${ }^{28-31}$

\section{Light scattering intensity measurements}

To measure the light scattered intensity, the solutions prepared in decalin and paraffin were put in test tubes and kept at 90 and $120^{\circ} \mathrm{C}$, respectively, for $5 \mathrm{~min}$ to avoid convection. The test tubes were immediately placed in a timeresolved light-scattering instrument, and measurements were performed at a desired temperature above $65^{\circ} \mathrm{C}$ using a $15 \mathrm{mV}$ helium-neon gas laser. In this instrument, six photodiodes were set at the desired scattering angle to simultaneously detect the scattering beam at various angles. The diameter of the light-scattering tube was $10 \mathrm{~mm}$, and the diameter of the irradiated spots of the laser beam was $1 \mathrm{~mm}$ to satisfy the Ergodic hypothesis.

SALS under cross-polarization (Hv) conditions was observed with a $3 \mathrm{mV}$ helium-neon gas laser as a light source. SALS patterns were observed for various times between the gelation and room temperatures.

\section{WAXD measurements}

WAXD measurements were carried out with a $12 \mathrm{~kW}$ rotating-anode X-ray generator (Rigaku RDA-rA, Akishima, Japan) operated at $150 \mathrm{~mA}$ and $40 \mathrm{kV}$. The X-ray beam using $\mathrm{CuK} \alpha$ radiation was monochromatized with a curved graphite monochrometer. Because of drastic viscosity increases in UHMWPE solutions at $1 \mathrm{~g}$ per $100 \mathrm{ml},{ }^{12} \mathrm{X}$-ray quartz cell tubes with 2.5 -mm diameter were used to pour very high viscosity UHMWPE solution into quartz cell tubes. Time resolution of the WAXD intensity was performed to study gelation mechanisms using a curved position-sensitive proportional counter to simultaneously estimate the change in diffraction intensity distribution as a function of the Bragg angle. The sample in the tube was heated to $145^{\circ} \mathrm{C}$ for $30 \mathrm{~min}$ and cooled quickly to the appropriate temperature.

\section{SAXS measurements}

SAXS measurements were carried out with a NanoStar U from Bruker (Karlsruhe, Germany). The X-rays were focused with a three-pinhole system with pinhole diameters of 500, 700 and $1000 \mu \mathrm{m}$. The optical bench length from the source to the sample was $1.5 \mathrm{~m}$, and the distance from the sample to the detector was $1.07 \mathrm{~m}$. This X-ray machine was equipped with a gas detector (Hi-Star) from Bruker with a $100 \mu \mathrm{m}$ pixel size. The accumulation time was $1 \mathrm{~h}$. The corrected intensity was obtained by subtracting the contribution of the background (corresponding to air scattering) from the total intensity.

\section{Differential scanning calorimetry (DSC) measurements}

DSC measurements were performed on $10 \mathrm{mg}$ gel samples sealed in aluminum pans with a DSC Q20 (TA Instruments, New Castle, DE, USA). In this experiment, the gel was packed in a sample pan without any space and heated to $150{ }^{\circ} \mathrm{C}$ for $>10 \mathrm{~min}$. The solution was cooled to room temperature, and the gel was reheated to $150^{\circ} \mathrm{C}$. The cooling and heating speeds were $1^{\circ} \mathrm{C}$ per min under $\mathrm{N}_{2}$ atmosphere.

\section{Polarized microscopy (POM) observations}

Gel and fibril structures were observed by polarized optical microscopy (Nikon OPTIPHOT 2-POL, Tokyo, Japan). POM images were observed for various times in the temperature range. To obtain POM images, a solution prepared at $150^{\circ} \mathrm{C}$ was poured into a small custom-made Petri dish, and the glass plate was covered to prevent solvent evaporation. The cell was placed on a heating plate with a temperature controller. The solution thickness was set through trial and error to record desirable POM images.

\section{Viscosity measurement}

Viscosities of decalin and paraffin solvents were measured using an AR2000ex rheometer (TA Instruments) at a fixed frequency of $1 \mathrm{rad} \mathrm{s}^{-1}$ while cooling from 150 to $20^{\circ} \mathrm{C}$. The UHMWPE solution viscosities in decalin and paraffin were measured from 130 to $70^{\circ} \mathrm{C}$. Before viscosity measurements, solutions were heated to $150{ }^{\circ} \mathrm{C}$ and cooled to $130{ }^{\circ} \mathrm{C}$, and measurements were started while cooling to avoid solvent evaporation during measurements between 150 and $130^{\circ} \mathrm{C}$.

\section{Viscoelastic properties}

The complex dynamic tensile modulus was measured at $10 \mathrm{~Hz}$ between 25 and $140^{\circ} \mathrm{C}$ using a viscoelastic spectrometer (VES-F) from Iwamoto Seisakusho Co., Ltd (Iwamoto, Japan). The specimen length between the jaws was $40 \mathrm{~mm}$ and the width was $\sim 3 \mathrm{~mm}$. These methods have been described in detail elsewhere. ${ }^{15}$

\section{RESULTS AND DISCUSSION}

\section{Viscosity and gelation time}

Figures 1a and b show the viscosities of the solvents and solutions as functions of temperature at $1 \mathrm{rad} \mathrm{s}^{-1}$. The viscosity of paraffin is slightly higher than decalin, but the difference is negligible relative to solution viscosities. The solution viscosity was much higher for decalin than paraffin at temperatures less than $\sim 120^{\circ} \mathrm{C}$. However, the gelation temperature of paraffin is higher than that of decalin in static state as shown in Figures $1 \mathrm{c}$ and d. These results seem to be incompatible. This paper focuses on the formation of ultradrawn crystal lamellae from two solvents with contradictory gelation properties.

Figures $1 \mathrm{c}$ and $\mathrm{d}$ show the gelation time with respect to temperature for the UHMWPE solutions prepared in decalin and paraffin, respectively, and quenched to the desired temperature. The 

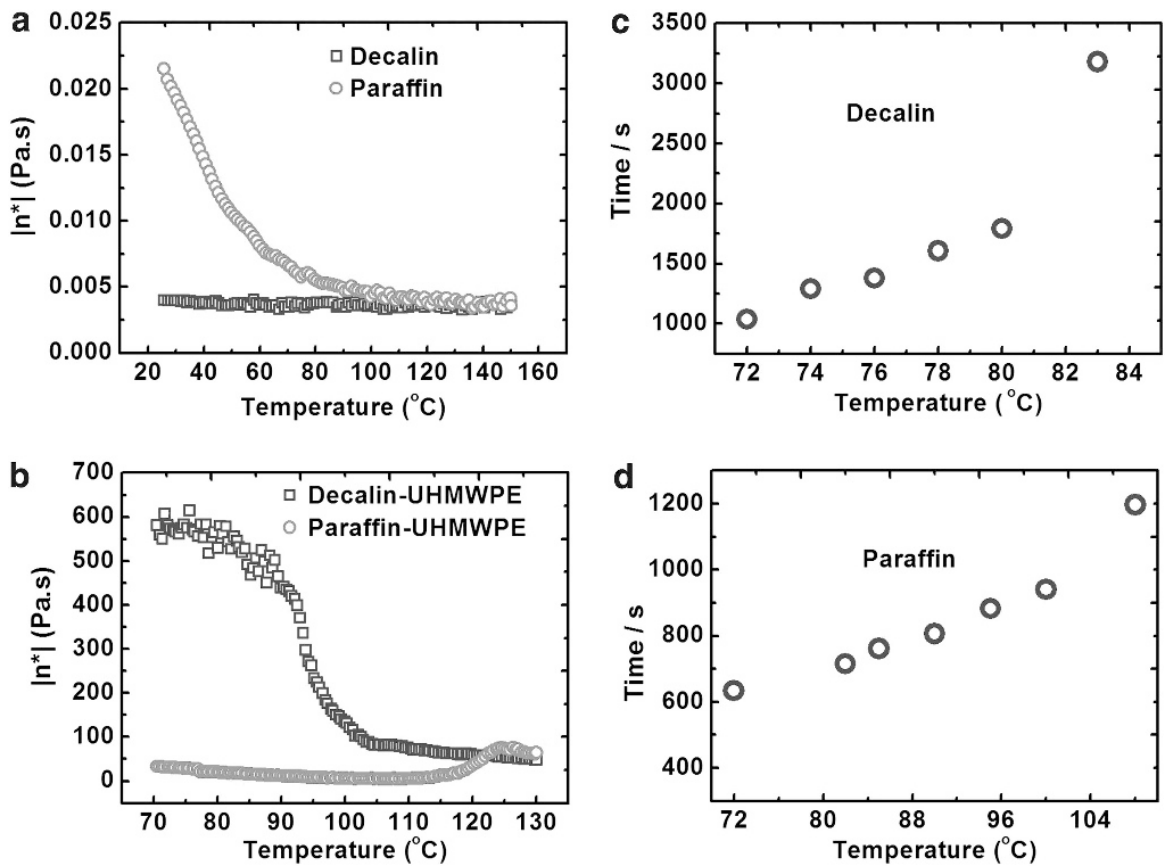

Figure 1 Viscosity versus temperature measured for (a) decalin and paraffin and (b) UHMWPE solutions prepared in decalin and paraffin, and temperature dependence of the gelation time for the UHMWPE solution prepared in (c) decalin and (d) paraffin. A full color version of this figure is available at the Polymer Journal online.
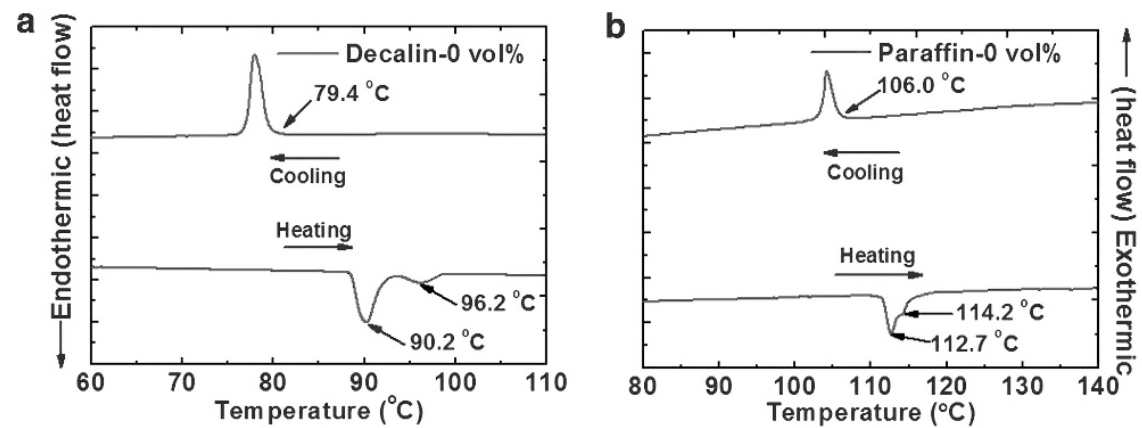

Figure 2 DSC curves of UHMWPE solutions prepared in (a) decalin and (b) paraffin. A full color version of this figure is available at the Polymer Journal online.

gelation time of UHMWPE chains was shorter in paraffin than decalin. The gelation time in decalin was $\sim 3250 \mathrm{~s}$ at $83^{\circ} \mathrm{C}$, and gelation did not occur beyond $83^{\circ} \mathrm{C}$, whereas gelation in paraffin at $83^{\circ} \mathrm{C}$ was achieved at $\sim 700 \mathrm{~s}$. Furthermore, the maximum for gel formation was $\sim 108^{\circ} \mathrm{C}$ and the gelation time was $\sim 1200 \mathrm{~s}$. These results indicated easy gelation/crystallization of UHMWPE chains in paraffin. When the solution paraffin solution was quenched, UHMWPE chain diffusion is thought to be less active and to accelerate the early formation of crosslinking points. The number of crosslinking points was larger in gels prepared in paraffin than in gels prepared in decalin when the UHMWPE in paraffin was quenched to $72-83^{\circ} \mathrm{C}$. This tendency at temperatures $<73^{\circ} \mathrm{C}$ was confirmed by WAXD as discussed below (Figures 5 and 6 ). Judging from the lower viscosity of the UHMWPE in paraffin in Figure 1b, UHMWPE chains formed comb-like structures by overlapping semiflexible chains to accelerate the local coagulation of UHMWPE chains, and the overlapping points were analogous to chemical crosslinks similar in swollen gels. The detailed discussion using a model is given below (Figure 11).
Figures 2a and b show DSC curves of UHMWPE solution prepared in decalin and paraffin, respectively. Measurements were performed while cooling from solution to gel (the first step) and heating from gel to solution (the second step). In Figure $2 \mathrm{a}$, crystallization started at $79.4^{\circ} \mathrm{C}$ in decalin, and the melting point corresponding to the endothermic peak appeared suddenly at $\sim 90.2^{\circ} \mathrm{C}$. For paraffin in Figure $2 \mathrm{~b}$, crystallization started at $\sim 106.0^{\circ} \mathrm{C}$ while cooling, and the melting point was $\sim 112.7^{\circ} \mathrm{C}$. In comparison with decalin, gelation in paraffin occurred at higher temperatures, which justified the shorter gelation time shown in Figures $1 \mathrm{c}$ and $d$.

The melting point corresponding to the endothermic peak changed drastically at $\sim 90.2^{\circ} \mathrm{C}$ in decalin and at $\sim 112.7^{\circ} \mathrm{C}$ in paraffin, indicating that the rapid ordering of UHMWPE chains in paraffin occurred at a higher temperature than in decalin because of the large number of coagulation points between chains. However, the area of the heat of fusion in decalin was larger than in paraffin, indicating larger crystallites. This finding suggests that the ordering of UHMWPE chains in decalin led to active liquid-liquid phase separation and that the formation of high-density polymer-rich phase 
a

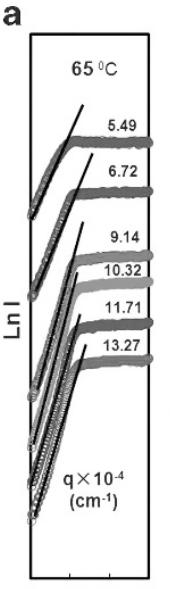

$0 \quad 100200300$ b

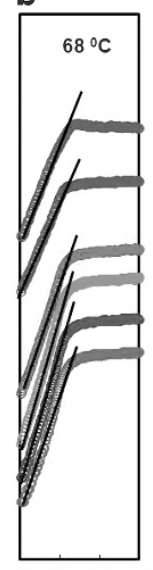

100200300 c

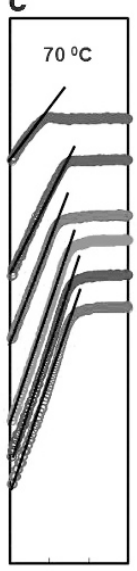

d

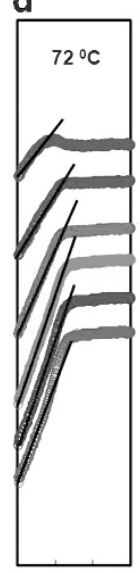

e

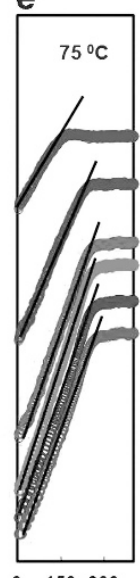

f

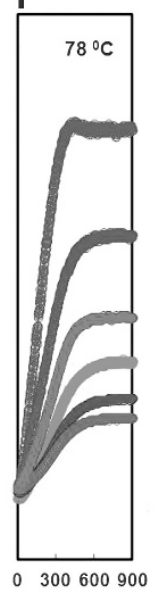

g

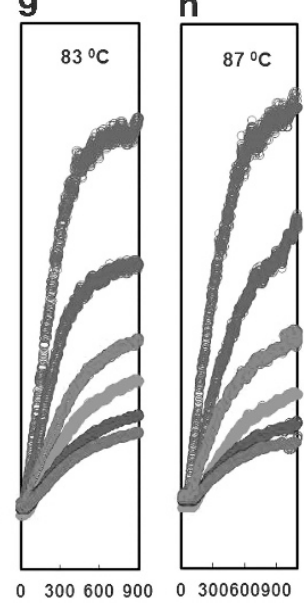

Time (s)

Figure 3 Change in the logarithmic plots $\ell n(l)$ of the scattered intensity against time at $q=\left(4 \pi n / \lambda^{\prime}\right) \sin (\theta / 2)$ for UHMWPE solutions prepared in decalin. a: $65^{\circ} \mathrm{C}$; b: $68^{\circ} \mathrm{C}$; c: $70^{\circ} \mathrm{C}$; d: $72^{\circ} \mathrm{C}$; e: $75^{\circ} \mathrm{C}$; f: $78^{\circ} \mathrm{C}$; g: $83^{\circ} \mathrm{C}$; $: 87^{\circ} \mathrm{C}$. A full color version of this figure is available at the Polymer Journal online.

areas accelerated with time. The formation of a high-density polymerrich phase in gels prepared in paraffin is less accelerated than in gels prepared by decalin. The crosslinking points in gels prepared in paraffin may resemble chemical crosslinks in the entire gel as discussed above.

\section{Phase separation and crystallization}

Time-resolved light scattered intensity measurements were carried out for UHMWPE solutions prepared in decalin and paraffin. Figures 3 and 4 show changes in the logarithmic plots of the scattered intensity against time at the indicated $q=\left(4 \pi n / \lambda^{\prime}\right) \sin (\theta / 2)$ for UHMWPE solutions prepared in decalin and paraffin, respectively, where $\lambda^{\prime}, \theta$ and $n$ are the wavelength of light in solution, the scattering angle and the refractive index (1.45) of the solvent, respectively. For the measurement of the scattered light intensity in decalin, the solutions were put in test tubes and kept at $90{ }^{\circ} \mathrm{C}$ for $2-3 \mathrm{~min}$ to avoid convection. The temperature drop was achieved within a few minutes.

The measurements in decalin were performed from 65 to $87^{\circ} \mathrm{C}$. To obtain reliable results, it was necessary to check the reproducibility by replicate experiments. As shown in Figure 3, the logarithm of the scattered intensity, $\ell n(I)$, increased linearly with time in the initial stage of phase separation and tended to level off, indicating a deviation from the linear relationship for crystalline polymer gels. ${ }^{28,30-34}$ In these figures, the plots at $t=0$ represented the beginning when the scattered intensity increased after setting the test tube in the light scattering instrument at the desired temperature. The deviation shifted to a shorter time scale, and the slope became steeper as the temperature decreased. At the leveling off point, the gel was opaque, indicating the beginning of crystallization of UHMWPE chains, which shall be discussed later. The leveling-off value of $\ell n(I)$ was constant beyond $1 \mathrm{~h}$ at temperatures below $75^{\circ} \mathrm{C}$. Beyond $78^{\circ} \mathrm{C}$, the slopes of $\ell n(I)$ became steeper with decreasing $q$, which was different than during the initial stage of phase separation. ${ }^{30-33}$ The linear increase in $\ell n(I)$ versus $t$ was observed up to $400 \mathrm{~s}$ at $87^{\circ} \mathrm{C}$, and the very gradual increase without leveling off was observed beyond $900 \mathrm{~s}$.

A similar tendency was observed for UHMWPE solution prepared in paraffin as shown in Figure 4. The measurements were performed at the indicated temperature after the solution was maintained at $120^{\circ} \mathrm{C}$ for $2-3 \mathrm{~min}$ to avoid convection. In comparison with decalin, the increase in $\ell n(I)$ against time $(t)$ could be observed beyond $95^{\circ} \mathrm{C}$, but at temperatures lower than $95^{\circ} \mathrm{C}$, the scattered intensity could not be detected because of gel opacity. The linearly increasing slope became less steep and extended over a longer period when the temperature was higher. At 108 and $111^{\circ} \mathrm{C}, \ell n(I)$ at $q<6.72 \times 10^{-4}$ $\left(\mathrm{cm}^{-1}\right)$ increased drastically up to ca. $280 \mathrm{~s}$ and decreased, whereas the gradual linear increase of $\ell n(I)$ at $q>6.72 \times 10^{-4}\left(\mathrm{~cm}^{-1}\right)$ was maintained beyond $400 \mathrm{~s}$ and leveled off. However, the linear slope was less steep with increasing $q$, which also differed from common behavior during the initial stage of phase separation associated with quasi-spinodal decomposition. ${ }^{30-33}$

A question arises as to whether crystal nuclei formed on the time scale of the linear increase in $\ell n(I)$ against $t$. If the linear relationship between $\ell n(I)$ and $t$ is related to liquid-liquid phase separation, then the deviation from the linear increase in $\ell n(I)$ against $t$ associated with the appearance of opaque color of the solution can be attributed to the formation of crystal nuclei during the later stage of phase separation, indicating the appearance of a polymer-rich phase. If this explanation is correct, then the initial stage is independent of the formation of crystal nuclei and X-ray diffraction peak can be detected only in the later stage.

Figure 5 shows WAXD intensity distributions from UHMWPE solutions prepared in decalin at the indicated temperatures, and experimental error was $\pm 0.5^{\circ} \mathrm{C}$. The insets on the right-hand side are enlargements of the curves around $21.6^{\circ}$, which show the time dependence of the peak heights. The curves at 0 and $400 \mathrm{~s}$ denote the accumulation of intensity from 0 to $400 \mathrm{~s}$ and $400-800 \mathrm{~s}$, respectively, because the accumulation period must be $400 \mathrm{~s}$ to ensure smooth diffraction curves. A negligible small diffraction peak appeared at $\sim 21.6^{\circ}$ (twice the Bragg angle), indicating the appearance of the (110) plane by a rapid temperature drop to $75^{\circ} \mathrm{C}$ in the time period 0-400 s, as shown in Figure 5a, because the time interval (0-150 s) associated with crystal nuclei growth (ensuring linear increase of $\ell n(I)$ with $t$ in Figure 3e) is too short to detect by WAXD. Even so, the very small peak at $75^{\circ} \mathrm{C}$ between 0 and $400 \mathrm{~s}$ can be attributed to diffraction in the 250-400 s range denoting deviations from a linear increase in $\ell n(I)$ against $t$ as shown in Figure 3e. In other words, the linearly increasing period $0-150 \mathrm{~s}$ corresponds to the initial stage of phase separation by chain diffusion to resolve an unstable thermodynamic state by quenching the solution. 
a

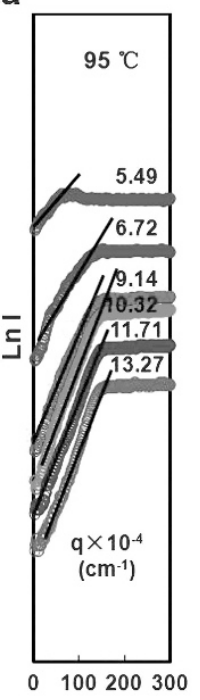

b

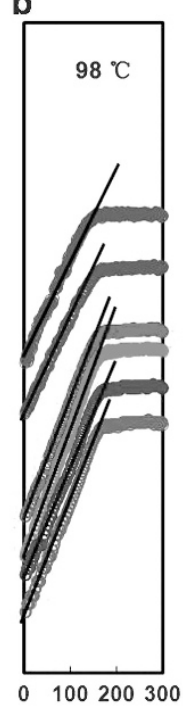

c

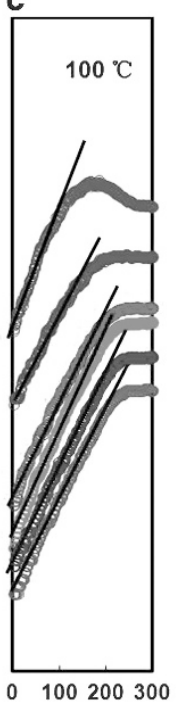

d

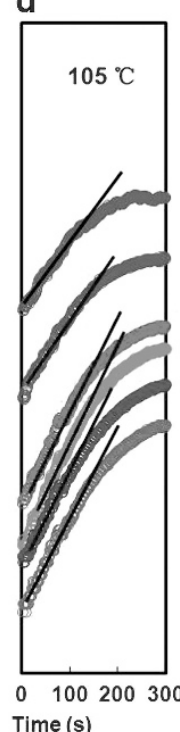

e

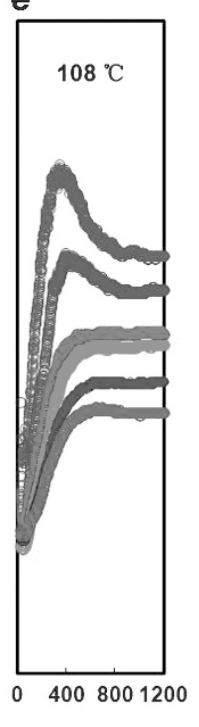

f

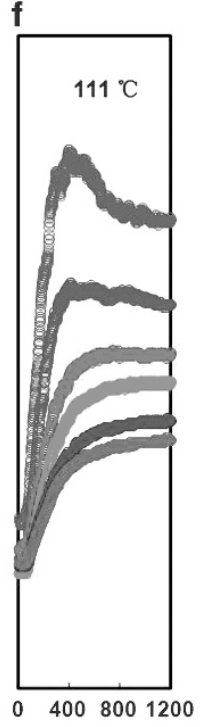

Figure 4 Change in the logarithmic plots $\ell n(l)$ of the scattered intensity against time at $q=\left(4 \pi n / \lambda^{\prime}\right) \sin (\theta / 2)$ for UHMWPE solutions prepared in paraffin. a: $95^{\circ} \mathrm{C}$; b: $98^{\circ} \mathrm{C}$; c: $100^{\circ} \mathrm{C}$; d: $105^{\circ} \mathrm{C}$; e: $108^{\circ} \mathrm{C}$; f: $111^{\circ} \mathrm{C}$. A full color version of this figure is available at the Polymer Journal online.

The diffraction curves at 75 and $78^{\circ} \mathrm{C}$ are almost identical. Apparently, crystallization by a rapid temperature drop to $83^{\circ} \mathrm{C}$ was not confirmed in the range 2000-2400 s. The appearance of local nuclei in the period $2400-2800 \mathrm{~s}$ shown in Figure $5 \mathrm{c}$ justifies the entire gelation at $3250 \mathrm{~s}$ shown in Figure 1c. The WAXD peak appeared later at elevated quenching temperatures. The peak intensity became slightly higher with time, as shown in Figures $5 \mathrm{a}-\mathrm{c}$, which is attributed to local appearance of crystal nuclei-like network structure. The development of loose folding structures with time plays an important role in gel formation.

Comparing the X-ray peak appearance period with $\ell n(I)$ against $t$ in Figure 3e, it is obvious that the small X-ray peak in the period $0-400 \mathrm{~s}$ at $75^{\circ} \mathrm{C}$ is attributed to the deviation from a linear increase in $\ell n(I)$ against $t$ in Figure 3e. A series of experiments on decalin solutions indicated that no crystallization occurred in the period of linearly increasing $\ell n(I)$.

Figure 6 shows X-ray diffraction data from UHMWPE solutions prepared in paraffin. The crystallization by a rapid temperature drop to $95^{\circ} \mathrm{C}$ occurred on a time scale of $0-400 \mathrm{~s}$, as shown in Figure 6a, although any peak did not appear for decalin at $87^{\circ} \mathrm{C}$, as shown in Figure $5 \mathrm{~d}$. In Figure 6b, the peak appeared at $105^{\circ} \mathrm{C}$ on the time scale of $0-400 \mathrm{~s}$, and the corresponding $\ell n(I)$ against $t$ leveled off as shown in Figure $4 \mathrm{~d}$. The peak height became more intense with time. In Figure $6 \mathrm{c}$, the peak at $108^{\circ} \mathrm{C}$ was negligibly small on the time scale of $0-400 \mathrm{~s}$, and it was not possible to attribute the peak to diffraction or noise, but the distinct peak at $800-1200 \mathrm{~s}$ corresponded to gel formation at $108^{\circ} \mathrm{C}$, as shown in Figure $1 \mathrm{~d}$. At $111^{\circ} \mathrm{C}$, no peak was visible even after $1 \mathrm{~h}$ in Figure 6d, indicating no gel formation as shown in Figure 1d. The very small X-ray peaks at 95 and $105^{\circ} \mathrm{C}$ in the time period $(0 \sim 400 \mathrm{~s})$ were attributed to the rapid formation of crystal nuclei, but the peak was confirmed to be slightly smaller than the other peaks beyond $400 \mathrm{~s}$, as the deviation from linearly increasing $\ell n(I)$ started beyond $c a .150 \mathrm{~s}$, as shown in Figures $4 \mathrm{a}$ and $\mathrm{d}$. Accordingly, it may be concluded that the formation of crystal nuclei did not appear during the period of linear increase $(0-150 \mathrm{~s})$ for the gel prepared by paraffin, and the same behavior was observed for the
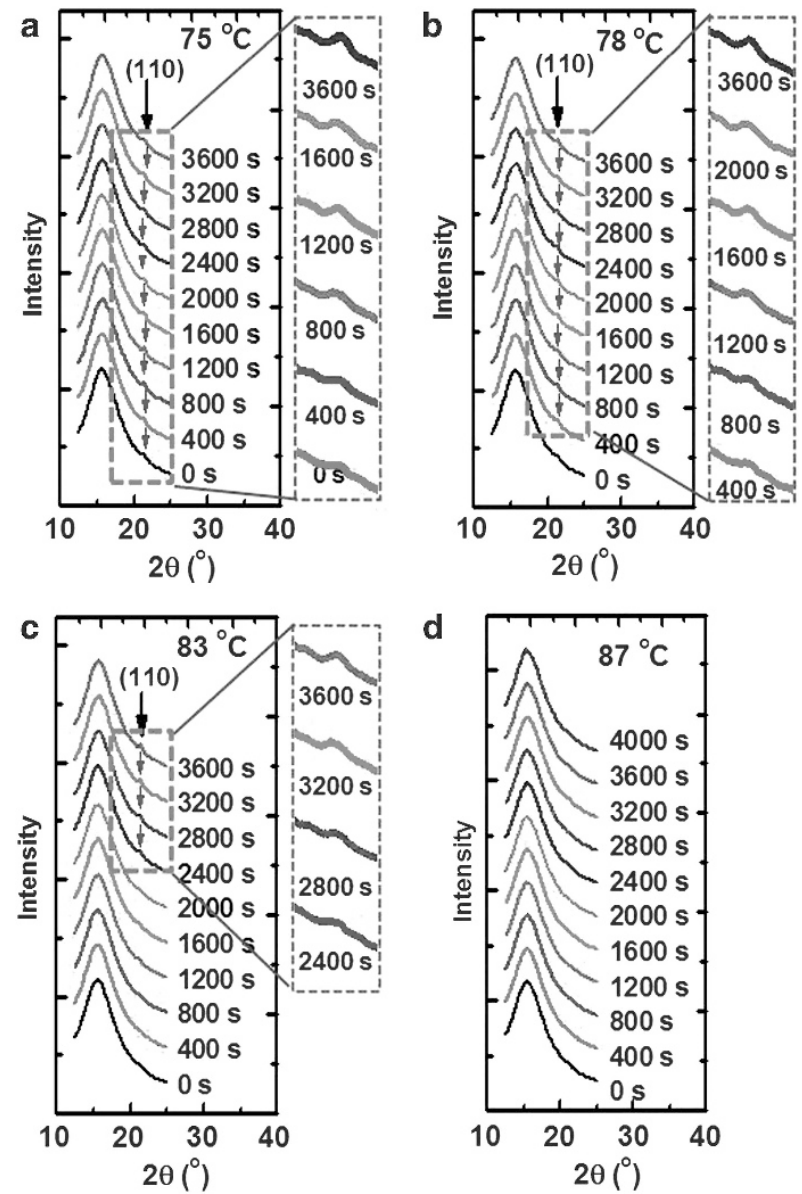

Figure 5 X-ray diffraction from UHMWPE solutions prepared in decalin at temperatures of (a) $75^{\circ} \mathrm{C}$, (b) $78{ }^{\circ} \mathrm{C}$, (c) $83^{\circ} \mathrm{C}$ and (d) $87^{\circ} \mathrm{C}$; the curve at $\mathrm{Os}$ and the curve at $400 \mathrm{~s}$ denote the accumulation of intensity in the range from 0 to $400 \mathrm{~s}$ and from 400 to $800 \mathrm{~s}$, respectively. A full color version of this figure is available at the Polymer Journal online. 

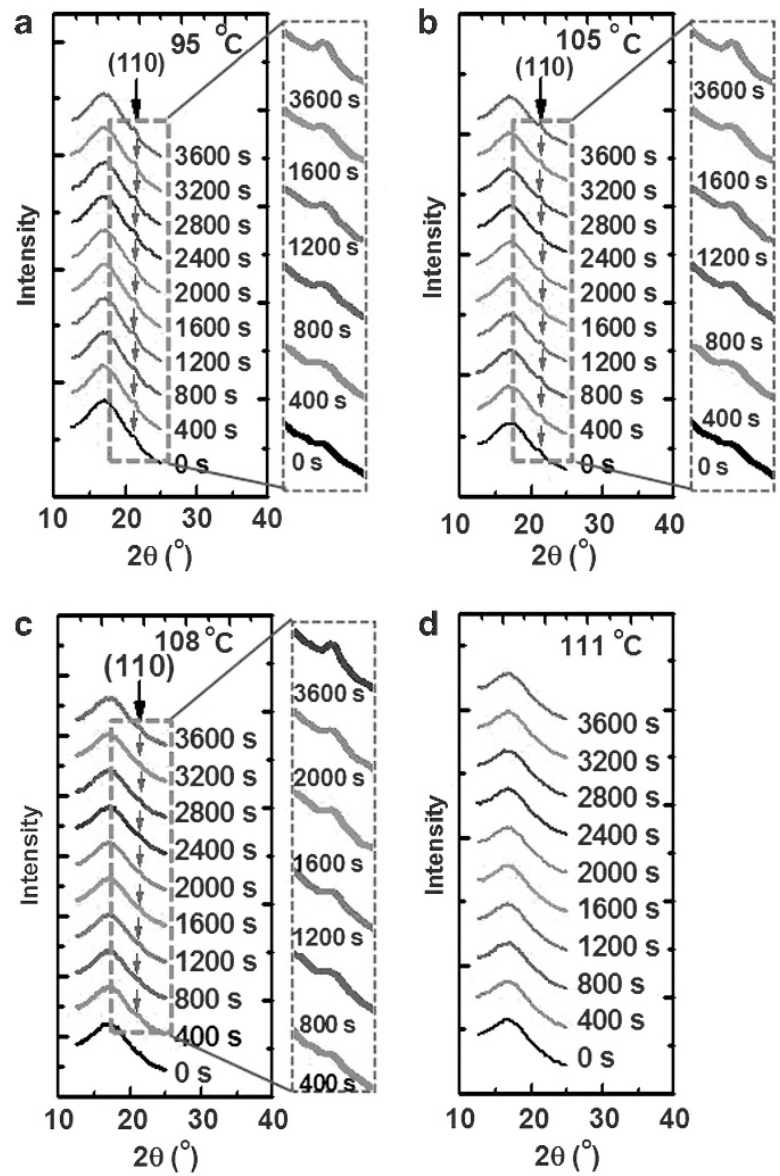

Figure 6 X-ray diffraction from UHMWPE solutions prepared in paraffin at temperatures of (a) $95^{\circ} \mathrm{C}$, (b) $105^{\circ} \mathrm{C}$, (c) $108^{\circ} \mathrm{C}$ and (d) $111^{\circ} \mathrm{C}$; the curve at $O s$ and the curve at $400 \mathrm{~s}$ denote the accumulation of intensity in the range from 0 to $400 \mathrm{~s}$ and from 400 to $800 \mathrm{~s}$, respectively. A full color version of this figure is available at the Polymer Journal online.

gel prepared in decalin. Although the experiments shown in Figures 5 and 6 could not be performed with synchrotron radiation, the small peaks were confirmed using a 12-kW rotating-anode X-ray generator (Rigaku RDA-rA) operated at $150 \mathrm{~mA}$ and $40 \mathrm{kV}$, and the measurements were repeated for the same solutions.

On the basis of the results in Figures 3-6, it may be concluded that no crystallization occurs in the initial stage of liquid-liquid phase separation of UHMWPE solutions. At temperatures $\leqslant 75^{\circ} \mathrm{C}$ for decalin and $\leqslant 105^{\circ} \mathrm{C}$ for paraffin, deviations from the linear relationship are similar to the later stage of spinodal decomposition in amorphous blends. As described before, this deviation shifts to shorter time scales as the temperature decreases. Accordingly, the linear increase in $\ell n(I)$ against time suggests the possibility of a successful analysis of the linear theory of spinodal decomposition proposed by $\mathrm{Cahn}^{43}$ and Cahn and Hilliard. ${ }^{43,44}$ If the linear relationship reflects the initial stage of spinodal decomposition as noted by Cahn ${ }^{43}$ then the change in scattered intensity in Figures 3-4 can be given by

$$
I(q, t)=I(q, t=0) \exp [2 R(q) t]
$$

where $I(q, t)$ is the scattered intensity at time $t$ after the initiation of the spinodal decomposition and $R(q)$ is the growth rate of concentration fluctuations as a function of $q$, which is given by

$$
R(q)=-D_{C} q^{2}\left\{\frac{\partial^{2} f}{\partial^{2} c}+2 \kappa q^{2}\right\}
$$

where $D_{c}$ is the translational diffusion coefficient of the molecules in solution, $f$ is the free energy of mixing, $c$ is the solution concentration and $\kappa$ is the concentration-gradient energy coefficient defined by Cahn and Hilliard. ${ }^{4}$

Figures $7 \mathrm{a}$ and $\mathrm{b}$ show the growth rate of concentration fluctuation $R(q)$ plotted against $q$ for the UHMWPE solutions in decalin and paraffin. The maximum growth rate $R\left(q_{\max }\right)$ increased with decreasing temperature $T$, and the value of the corresponding scattering vector, $q_{m}$, was maintained at the same angle. This phenomenon is in good agreement with the principle of spinodal decomposition in amorphous polymer solutions proposed by van Aartsen, ${ }^{45}$ provided the spinodal temperature $T_{s}$ is higher than $75^{\circ} \mathrm{C}$ for decalin and $105^{\circ} \mathrm{C}$ for paraffin. The growth of concentration fluctuations during the initial stage promotes the molecular aggregation and crystallization (appearance of crystal nuclei) in the later stage. However, the spinodal ring did not appear because of the dull scattering maximum. To observe the ring, the peak must be two or three orders of magnitude greater. Judging from the curves in Figures $7 \mathrm{a}$ and $\mathrm{b}$, the concentration fluctuations by UHMWPE chain diffusion in decalin in the temperature range $65-75^{\circ} \mathrm{C}$ are larger than in paraffin in the temperature range $95-105^{\circ} \mathrm{C}$. This means that $R(q)$ is not closely related to solution viscosity but is associated with the coagulation speed of UHMWPE chains to form gels. Accordingly, the period of the initial stage becomes shorter with increasing concentration and, consequently, network formation and crystallization occur rapidly in the later stage. Similar behavior was confirmed for UHMWPE with $\bar{M}_{v}=6 \times 10^{-6} .32$

More conclusive evidence for quasi-spinodal decomposition is based on the linear relationship. The quasi-spinodal temperature $T_{s}$ was estimated according to the linear theory. Plots of $R(q) / q^{2}$ vs $q^{2}$ were prepared at the measured temperature. The plots showed a linear relationship, and the apparent diffusion coefficient $D_{\text {app}}$, defined by $D_{\text {app }}=D_{c}\left(\partial^{2} f / \partial c^{2}\right)$, can be obtained from the intercept on the vertical axis.

The values of $D_{\mathrm{app}}=-D_{c}\left(\partial^{2} f / \partial c^{2}\right)$ are significant. Because of the positive values of $D_{c}$, the values of $\partial^{2} f / \partial c^{2}$ took a negative value, characterizing unstable regions and leading to spinodal decomposition. Figures 7c and d show the temperature dependence of $D_{\text {app }}$ for the UHMWPE solutions prepared in decalin and paraffin, respectively. A fairly good linear relationship was obtained. From the intercept on the temperature axis, the quasi-spinodal temperature $T_{s}$ at $D_{\text {app }}=0 . T_{s}$ of UHMWPE was higher in paraffin than in decalin. The higher $T_{s}$ in paraffin justifies the higher gelation temperature compared with decalin. This indicates that UHMWPE chains in paraffin rapidly formed crosslinking points to accelerate gelation at high temperatures $\geqslant 95^{\circ} \mathrm{C}$. In contrast, as shown in Figures $7 \mathrm{a}$ and $\mathrm{b}$, the growth rate $R(q)$ of concentration fluctuations in decalin was faster than in paraffin. This difference is because of the fact that the liquid-liquid phase separation in decalin was accelerated with time, as $T_{s}\left(88^{\circ} \mathrm{C}\right)$ for decalin was lower than $T_{s}\left(109^{\circ} \mathrm{C}\right)$ for paraffin.

However, the time dependence of the phase separation could not be determined on the basis of the results in Figures 3-6 because the time dependence of the amorphous X-ray peak of UHMWPE and the conversion from gauche- to trans-conformations by time-resolved Fourier transform infrared spectroscopy were impossible to resolve because of the very large scattering peaks from the solutions and the very low concentrations of UHMWPE. 

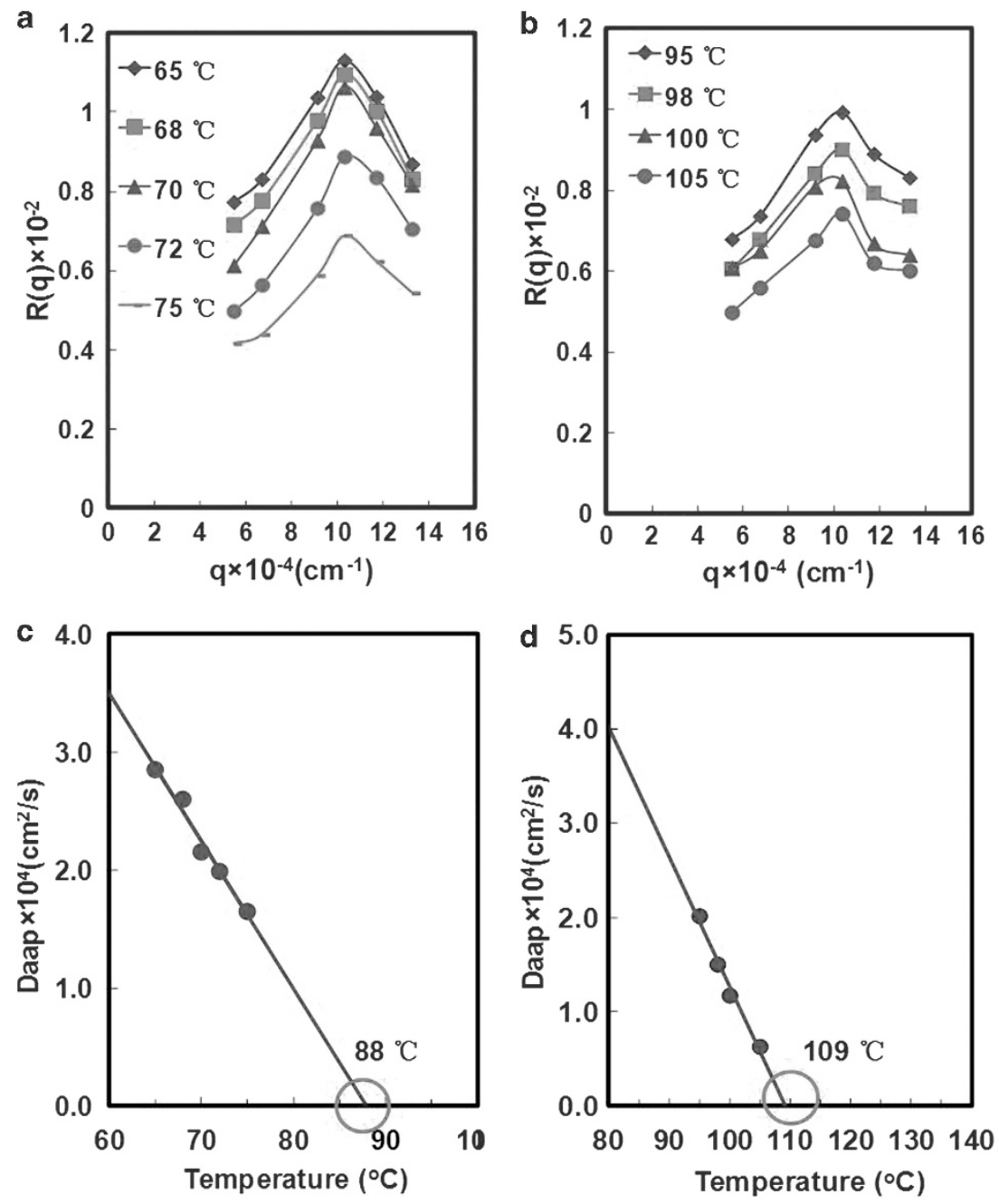

Figure 7 Growth rate $R(q)$ of concentration fluctuation plotted against $q$ for the UHMWPE solution prepared in (a) decalin and (b) paraffin. Temperature dependence of $D_{a p p}$ for the UHMWPE solution prepared in (c) decalin and (d) paraffin. A full color version of this figure is available at the Polymer Journal online.

Returning to Figure 2, DSC curves during the cooling for gelation of UHMWPE solutions prepared in decalin and paraffin are in good agreement with the light scattering and X-ray results. Chain diffusion, phase separation and the formation of crystal nuclei occurred at lower temperature in decalin than in paraffin. However, exothermic peak areas are smaller in paraffin than in decalin, indicating less effective crystallite growth. The reason can be justified by SALS patterns under Hv polarization conditions and the corresponding POM images.

Figure 8 shows changes in $\mathrm{Hv}$ patterns with time at 75, 70 and $65^{\circ} \mathrm{C}$ lower than $T_{s}\left(88^{\circ} \mathrm{C}\right)$. To facilitate understanding, the relationship between $\ell n(I)$ and time at $q=6.72 \times 10^{-4} \mathrm{~cm}^{-1}$ in Figure 3 is shown again in this figure. In the initial stage corresponding to the linear increase in $\ell n(I)$ vs $t$, the Hv scattering provided no patterns. At the beginning of the later stage $\left(45 \mathrm{~s}\right.$ at $\left.65^{\circ} \mathrm{C}\right)$, an indistinct circular pattern was observed, indicating weak chain coagulation in the polymer-rich phase. With time, the patterns at 75 and $90 \mathrm{~s}$ showed a slightly clearer X-type reflection correlation distance of anisotropic elements in three-dimensional space, and the pattern at $180 \mathrm{~s}$ shows a clear X-like scattering from rod-like structures. This is because of the appearance of sites with dense-chain coagulation of optically anisotropic elements in the polymer-rich phase. By the progression of dense-chain coagulation with time, the electron density of the polymer-rich phase becomes higher, and crystallite sizes become larger by forming lamella structures. The corresponding POM images at 45,75 and 90 s showed the presence of very indistinct structures in dark version. Incidentally, similar indistinct X-type patterns were also observed in the later stage at 70 and $75^{\circ} \mathrm{C}$.

On the other hand, the Hv scattering from gels prepared in paraffin showed no patterns and/or a very indistinct circular type pattern that was independent of time and temperature, in spite of the crystal nuclei formation at higher temperature than decalin. Even by quenching the solution to room temperature, very little gel syneresis occurred, but most of the paraffin was maintained in the gel, and the crystal growth was much slower than in gels containing decalin.

Thus, the syneresis of gels prepared by decalin was accelerated with time, and the outflow of the solvent stimulated crystal growth when quenched to room temperature $\left(30^{\circ} \mathrm{C}\right)$, whereas gel syneresis by quenching was very slight in paraffin. To demonstrate this phenomenon, X-ray measurements were carried out after quenching to $30^{\circ} \mathrm{C}$ in both solutions. Figure $8 \mathrm{~b}$ shows diffraction curves obtained $1 \mathrm{~h}$ after the quenching. Both curves show the same peak heights of (110) at $21.6^{\circ}$ and (200) planes at $24.0^{\circ}$. After 5 days, however, the 

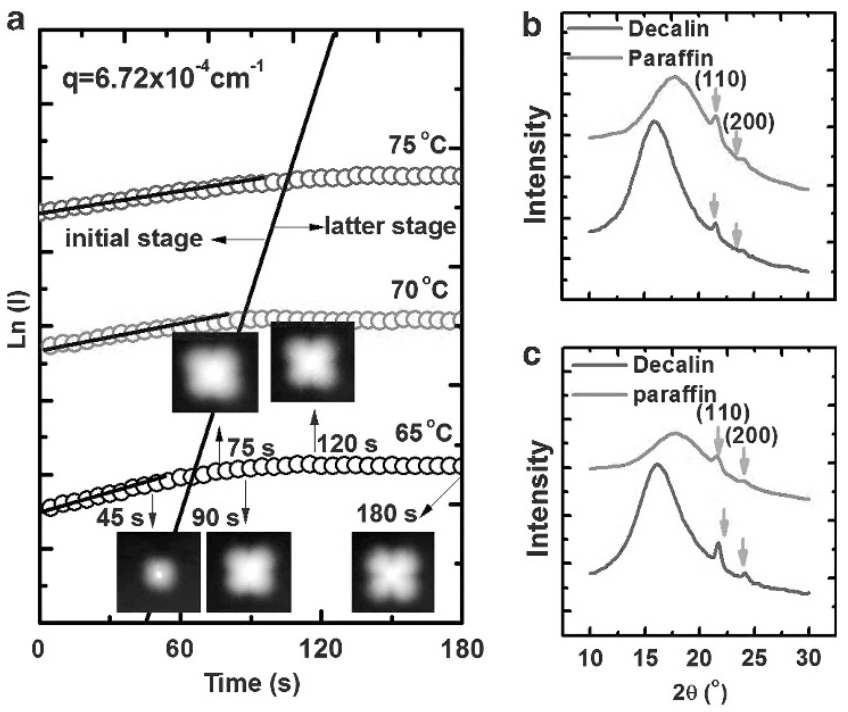

Figure 8 Time dependence of $\mathrm{Hv}$ patterns at $65^{\circ} \mathrm{C}$, observed at the scattered intensity $(\ell n(/))$ on time at $q=6.72 \times 10^{-4} \mathrm{~cm}^{-1}$, and X-ray diffraction curves of gels prepared by quenching solutions to $30^{\circ} \mathrm{C}$; measurements were performed immediately for (b) gels prepared by decalin and paraffin. (c) Measurements were performed after 5 days for gels prepared in decalin and paraffin. The accumulation time for all the measurements was $1 \mathrm{~h}$. A full color version of this figure is available at the Polymer Journal online.

diffraction peak heights from gels containing decalin became much more intense, whereas the two peaks for gels containing paraffin maintained the same diffraction heights, as shown in Figure 8c. This difference is attributed to the different crystal growths in the gels prepared in decalin and paraffin.

Furthermore, the difference in peak height became more remarkable each day because of the progression of gel syneresis containing decalin. This progression was thought to be because of the chemical structure of decalin, which is quite different from that of $\mathrm{PE}$, whereas the chemical structure of paraffin is similar to that of PE. Hence, good affinity between UHMWPE and paraffin provided rapid formation of crystal nuclei at high temperature such as $108^{\circ} \mathrm{C}$, but paraffin was maintained in the gel for a long time after quenching to room temperature. Good affinity of paraffin hampered the growth of original crystal nuclei in polymer-rich phases. In contrast, poor affinity between $\mathrm{PE}$ and decalin hampered crystal nuclei at high temperatures such as $95^{\circ} \mathrm{C}$. However, at temperatures lower than $T_{s}$, active chain diffusion by liquid-liquid phase separation provided polymer-rich phases, and the electron density of the polymer-rich phase became higher with time. Accordingly, the indistinct pattern changed into a clear X-type pattern with time as shown in Figure 8a. On the other hand, the $\mathrm{Hv}$ patterns from gels prepared in paraffin showed no scattering or extremely an indistinct circular type. These profiles were maintained beyond 1 month, even when solutions were quenched to room temperature. The gels prepared in decalin were in a nonequilibrium state, whereas the gels prepared in paraffin were in a stable state.

To obtain further conclusive evidence, WAXD and SALS results were confirmed by POM observations. Figure 9a shows POM images for gels prepared in decalin quenched to $87^{\circ} \mathrm{C}$ (near $T_{s}=88^{\circ} \mathrm{C}$ ) from $145^{\circ} \mathrm{C}$. These images were taken with a $1 / 4$-wavelength plate to detect structures. In the time interval of $0-800 \mathrm{~s}$, the POM image showed a dark image, and the $\mathrm{Hv}$ scattering showed no pattern, indicating a lack of correlation between anisotropic optical elements. ${ }^{34}$ In the period from $900 \mathrm{~s}$ ( $15 \mathrm{~min})$ to $5400 \mathrm{~s}$ ( $90 \mathrm{~min})$, the POM image showed continuous structures, but faint yellow and blue colors characterizing optical anisotropy were observed locally under crosspolarization conditions (no 1/4-wavelength plate). The corresponding $\mathrm{Hv}$ scattering provided an indistinct circular pattern that was much less distinct than those at $45 \mathrm{~s}$ at $65^{\circ} \mathrm{C}$ in Figure 8a, indicating very weak correlation distances between optical elements. ${ }^{34}$ Judging from Figures $1 \mathrm{c}$ and $5 \mathrm{c}$, the unclear images after $900 \mathrm{~s}(15 \mathrm{~min})$ and $5400 \mathrm{~s}$ (90 min) under POM observation at $87^{\circ} \mathrm{C}$ showed structures with no crystal nucleus during gelation.

Figure $9 \mathrm{~b}$ shows POM images for UHMWPE solutions prepared in paraffin. The photos were taken at 0 and $90 \mathrm{~min}$ after quenching the solutions to $107^{\circ} \mathrm{C}$. The POM images reveal the presence of an unclear continuous network at $107^{\circ} \mathrm{C}$, although the POM image at $87^{\circ} \mathrm{C}$ from a gel prepared in decalin provided dark images, as shown in Figure 9a. Interestingly, the POM image of a gel prepared in paraffin at $107^{\circ} \mathrm{C}$ persisted even after $5400 \mathrm{~s}$ (90 min), but $\mathrm{Hv}$ scattering showed no pattern. The WAXD intensity shown in Figure $6 \mathrm{c}$ suggests the existence of crystal nuclei. Furthermore, the POM image from gels at $87^{\circ} \mathrm{C}$ in Figure 9c revealed the same continuous structure as the gels prepared at $107^{\circ} \mathrm{C}$, and the structures were maintained after $5400 \mathrm{~s}$ ( $90 \mathrm{~min}$ ). A similar image was also observed even for the gel quenched to room temperature. Interestingly, the WAXD intensity in Figures 5 and 6 indicated that the very small continuous structures for paraffin at 107 and $87^{\circ} \mathrm{C}$ contained crystal nuclei. In contrast, the slightly clearer continuous structure of gel in decalin at $900 \mathrm{~s}$ at $87^{\circ} \mathrm{C}$ contained no crystal nuclei, as shown in Figure $5 \mathrm{~d}$. The corresponding $\mathrm{Hv}$ patterns in Figure 9 showed much less distinct detail than the circular pattern at $45 \mathrm{~s}$ at $65^{\circ} \mathrm{C}$ in Figure $8 \mathrm{a}$, indicating very weak correlation distance between the optical elements.

The POM observations in Figure 9 justified the light scattering and $\mathrm{X}$-ray results in Figures 3-8. The phase separation of gels prepared in decalin increased over time at temperatures lower than $T_{s}$. In contrast, gels prepared in paraffin formed crystal nuclei at $107^{\circ} \mathrm{C}$, but such conditions were achieved within $400 \mathrm{~s}$, and no $\mathrm{Hv}$ patterns were observed for the gels at $87^{\circ} \mathrm{C}$ (much lower than $T_{s}=108^{\circ} \mathrm{C}$ ) for $90 \mathrm{~min}$.

In contrast, for decalin, changes in $\mathrm{Hv}$ scattering patterns were sensitive to time at temperatures such as $65^{\circ} \mathrm{C}$, which was much lower than $T_{s}\left(88^{\circ} \mathrm{C}\right)$. The observed $\mathrm{Hv}$ patterns can be classified into three types, unclear circular type, unclear X-type and clear X-type, as shown in Figure $8 \mathrm{a}$. The mechanism has been analyzed elsewhere. ${ }^{34}$

These phenomena can be summarized as follows. According to the previous results, ${ }^{34}$ polymer-rich and polymer-poor phases during liquid-liquid phase separation are optically isotropic in the initial stage. Over time, small lamellae with defects (that is, not crystalline) corresponding to optically anisotropic elements appeared locally in the polymer-rich phase, but the polymer-rich phase was still optically isotropic, as discussed elsewhere. ${ }^{34}$ As for gels prepared in paraffin, the progression of the phase separation did not occur because there was no gel syneresis. The Hv scattering provided no patterns at high temperatures around $107^{\circ} \mathrm{C}\left(T_{s}=109^{\circ} \mathrm{C}\right)$, and it provided an indistinct circular type of pattern for quenching to room temperature, which can be calculated theoretically ${ }^{34}$ using a statistical approach in three-dimensional space. Such an indistinct circular pattern could be achieved using parameters with weak correlation distances for polar and azimuthal angles between the two principle axes of two anisotropic optical elements. On the other hand, phase separation was developed for gels prepared in decalin. In this case, the two 


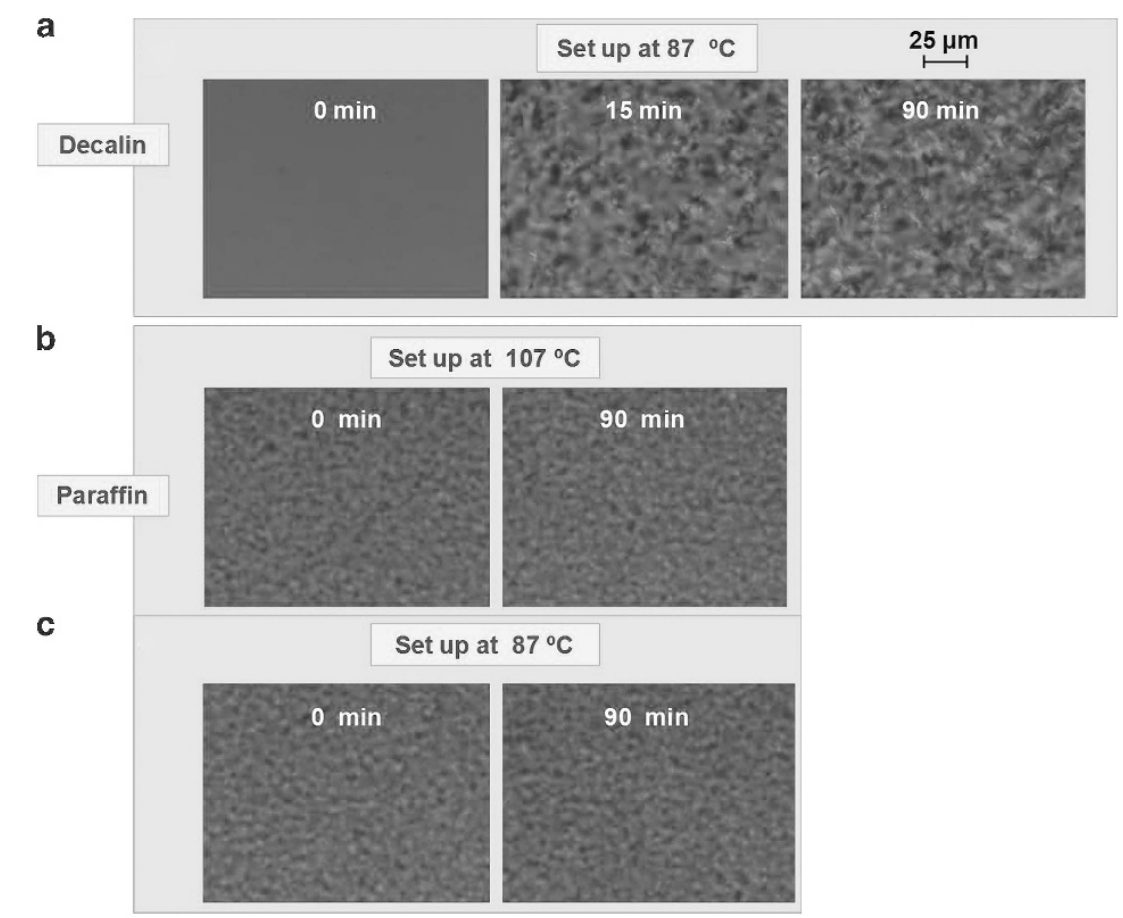

Figure 9 POM images for gels observed after quenching solutions to the indicated temperatures. The POM images were taken with a 1/4-wavelength plate. (a) Quenching gel prepared in decalin at $87^{\circ} \mathrm{C}$. The images were taken at 0,15 and $90 \mathrm{~min}$. (b) Quenching gel prepared in paraffin at $107^{\circ} \mathrm{C}$. The images were taken at 0 and $90 \mathrm{~min}$. (c) Quenching gel prepared in paraffin at $87^{\circ} \mathrm{C}$. The images were taken at 0 and 90 min. A full color version of this figure is available at the Polymer Journal online.

optically anisotropic elements have a distance correlation, as free movements between the two elements are restricted. This calculation by selecting parameters for the correlation distance provided an indistinct circular-type pattern at $45 \mathrm{~s}$ at $65^{\circ} \mathrm{C}$ in Figure $8 \mathrm{a}$. With further development of the phase separation, the small lamellae with defects in the polymer-rich phase were closer, and the movement of the neighboring elements was strongly restricted. In this case, the $\mathrm{Hv}$ scattering provided an X-type pattern. A clear X-type pattern appeared at $90 \mathrm{~s}$ at $65^{\circ} \mathrm{C}$, as shown in Figure 8a. Such an Hv pattern can be calculated by selecting parameters with strong correlation distances for the polar and the azimuthal angles between the two principle axes of two anisotropic optical elements. This mathematical treatment is much more complicated than the method proposed by Stein and Wilson. ${ }^{46}$

Over time, a clear X-type pattern appeared, as shown in Figure 8a at $180 \mathrm{~s}$ at $65^{\circ} \mathrm{C}$, because of further development of the phase separation. In this case, the $\mathrm{Hv}$ pattern provided a clear X-type pattern with four very clear lobes. Such scattering is attributed to optically anisotropic rods that can be constructed by coagulating the anisotropic scattering elements associated with a loosely folded structure.

The calculation was carried out using a statistical approach, which is different from the well-known $\mathrm{Hv}$ scattering from solid-state studies. ${ }^{47,48}$ For instance, a stiff gel implies that the boundaries of the polymer aggregation structures in the gels become clearer with time because the distance correlation between optically anisotropic elements in the polymer-rich phase becomes stronger locally in the latter stage, whereas the correlations in the polymer-poor region become weaker as in a dilute solution. In such a case, the correlation distance is be limited to length $L$ corresponding to the length of a rod-like structure. This is the result of assuming the rod to be infinitesimally thin in diameter ${ }^{34}$ to achieve good agreement between observed and calculated patterns.

As discussed in Figures $8 \mathrm{~b}$ and $\mathrm{c}$, the gels prepared in decalin provoked syneresis. The gel syneresis was accelerated with time, and the outflow of the solvent by syneresis stimulated crystal growth. If the solution was poured into a Petri dish, the dry gel film can be obtained by allowing decalin to flow out from the wet gel, where the gel syneresis occurs mainly in the direction of the film thickness because of surface adhesion between the gel and the bottom of the glass Petri dish. On the other hand, the gel syneresis prepared in paraffin was extremely small, as discussed above, and drying was achieved by forcibly covering the gel surface with filter paper. By changing the filter paper, the paraffin was removed gradually from gel by blotting. Then, paraffin residues were removed in hexane.

\section{Lamellar structure and ultradrawing}

The crystallinity volume in the dry gel films prepared in decalin and paraffin were $76.5 \%$ and $73.2 \%$, respectively, which were estimated by assuming the densities of the crystal and the amorphous phases to be 1.00 and $0.852 \mathrm{~g} \mathrm{~cm}^{-3}$, respectively.

Figures $10 \mathrm{a}$ and $\mathrm{b}$ show the SAXS intensity distributions, and Figures $10 \mathrm{c}$ and $\mathrm{d}$ show patterns (edge) for dried gel films prepared in decalin and paraffin, respectively, with the X-ray beam directed parallel to the film surface. The intensity distributions in Figures $10 \mathrm{a}$ and $\mathrm{b}$ were measured in the vertical direction of the patterns in Figures 10c and d. That is, the left- and right-side results show the intensity distributions and the patterns for decalin and paraffin dry gel films, respectively. The SAXS intensity and the corresponding patterns showed three-order scattering maxima. The experimental and the theoretical curves are shown in red and blue. The theoretical curves were calculated using models in Figures $10 \mathrm{~g}-\mathrm{i}$. 

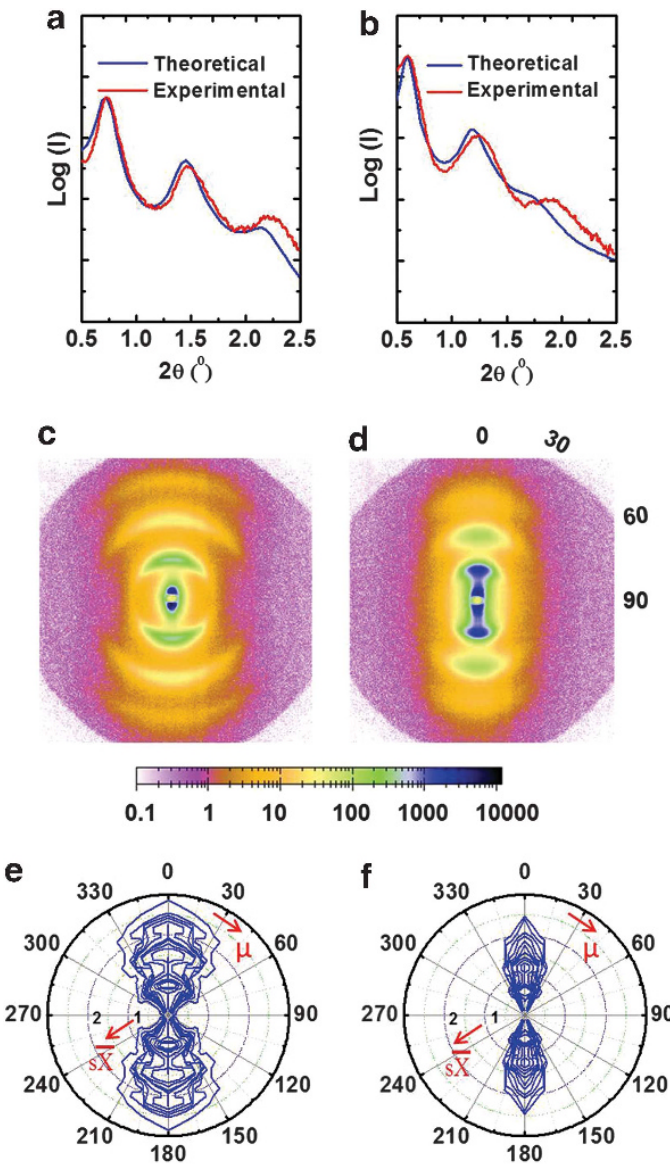
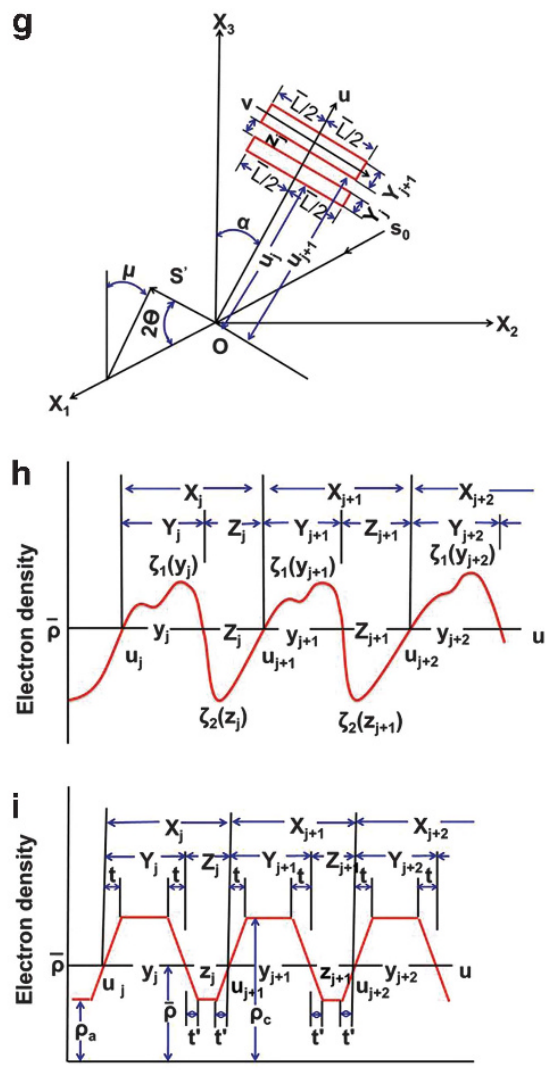

Figure 10 SAXS intensity in the vertical direction. (a) Dry gel films prepared in decalin and (b) dry gel films prepared in paraffin. (c) SAXS patterns (edge) from a decalin film; (d) SAXS image patterns from a paraffin film; (e) theoretical SAXS patterns from a decalin film; (f) theoretical SAXS patterns from a paraffin film. (g) Coordinates to calculate SAXS intensity distributions from lamellar assemblies, where the $X_{3}$ axis corresponds to the film normal direction and the $X_{1}$ axis is parallel to the direction of an incident beam whose unit vector is defined as $\boldsymbol{s}_{0}$. (h) Density variation of two phases by positive and negative deviations from the average density of the system represented by an arbitrary function; (i) density variation represented by a trapezoidal function. ${ }^{37}$

Incidentally, the WAXD patterns (not shown in this paper) showed preferential orientation of the c-axes for both films, as already observed elsewhere. ${ }^{2,7,8}$ The SAXS and WAXD patterns indicated that the dried gel films were composed of crystal lamellae that were highly oriented with their large flat faces parallel to the film surface but randomly oriented with respect to the direction normal to the film surface, and within the crystal lamella, the c-axes are oriented perpendicular to the large flat faces. Thus, when the as-cast UHMEPE gel films are dried by slow evaporation of the solvent, the lamellar crystals orient parallel to the film surface in a manner similar to mats of single crystals. ${ }^{2,9}$ As illustrated in the SAXS intensity, the scattered intensity distribution with the third-order scattering maxima can be observed indicating high-order orientation of stacking crystal lamellae. The identity periods for the dry gel films prepared in decalin and paraffin were 12.1 and $14.7 \mathrm{~nm}$, respectively, which were estimated by the first-order peak.

To pursue the detailed discussion for the lamellar arrangement on the two-dimensional plane through theoretical calculations, models in which the density variation of the two phases is specified by positive and negative deviation from the average density of the system, as in neutron scattering, were proposed, and they are illustrated in Figures 10g-i. The outline of the theoretical calculation to explain parameters has been described in the Appendix, and the detailed mathematical derivation has been discussed elsewhere. ${ }^{37}$ The theoretical curves in blue show the SAXS intensity distribution along the vertical direction of the SAXS images. The theoretical curves were normalized by equation (A8) in the Appendix, and then the heights of the first-order peak top were shifted vertically to overlap the experimental first peak height because the peak profiles of theoretical calculation are not affected by this treatment. This procedure is suitable for comparing experimental curves with theoretical curves by parameter setting.

The theoretical curves calculated by parameter fitting are in good agreement with the experimental curve. That is, the parameters by computer simulation are as follows:

Decalin

$$
\begin{aligned}
& \bar{Y} / \bar{L}=0.02, \bar{X} / \bar{L}=0.03, \sigma_{y} / \bar{Y}=0.11, \sigma_{z} / \bar{Z}=0.11, \quad \bar{N}=5, \\
& \sigma_{N}=3, \\
& \sigma_{\alpha}=3, t=0.051, t^{\prime}=0.166 \\
& \text { Paraffin } \\
& \bar{Y} / \bar{L}=0.02, \bar{X} / \bar{L}=0.03, \sigma_{y} / \bar{Y}=0.15, \sigma_{z} / \bar{Z}=0.15, \quad \bar{N}=8, \\
& \sigma_{N}=3, \\
& \sigma_{\alpha}=5, t=0.047, t^{\prime}=0.129
\end{aligned}
$$

As shown in Figures $10 \mathrm{~g}-\mathrm{i}, \bar{Y}$ and $\bar{Z}$ are the average values of the representative phases with fluctuation, $\bar{X}$ is the average lamellar length (identity period) given by $\bar{X}=\bar{Z}+\bar{Y}$, and $\sigma_{y}$ and $\sigma_{z}$ are standard deviations. The experimental values of $\bar{X}$ for decalin and paraffin were given as 12.1 and $14.7 \mathrm{~nm}$, respectively. 

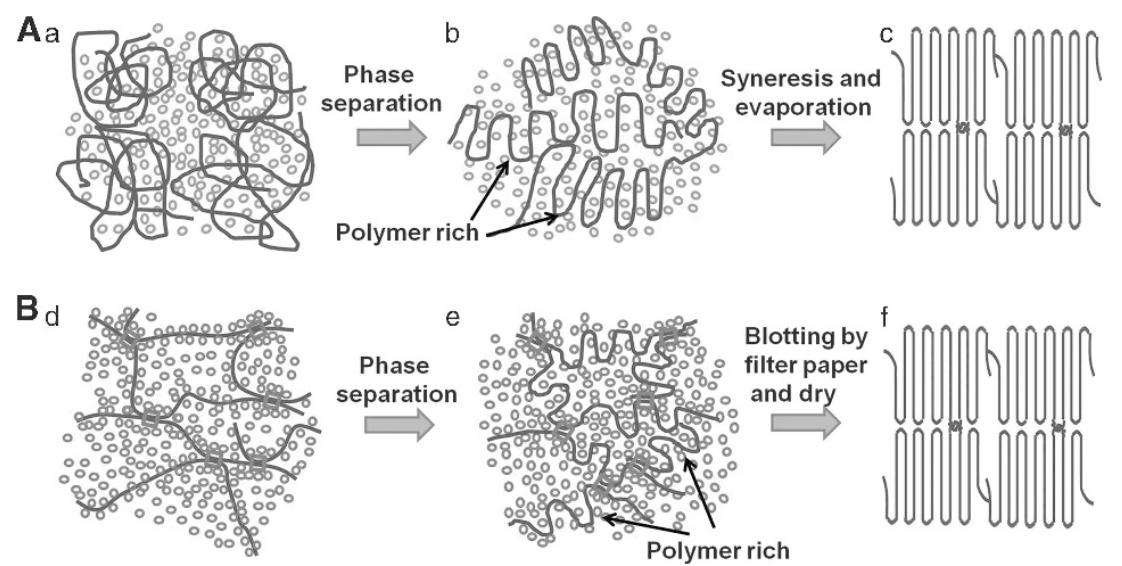

Figure 11 Models (a-f) representing gelation mechanisms and formation of lamellae under drying processes for UHMWPE chains in (A) decalin and (B) paraffin. A full color version of this figure is available at the Polymer Journal online.

According to the general numerical calculations, ${ }^{37}$ the values of $\sigma_{y} / \bar{Y}$ and $\sigma_{z} / \bar{Z}$ in equations (A14) and (A15) in the Appendix are very sensitive to the peak profile and $\bar{X} / \bar{L}$ is unknown parameter. However, it was confirmed that profile changes in SAXS patterns are not sensitive to $\bar{X} / \bar{L}$ in the range from 0.1 to 0.02 (see Figures 10 and 11 in Matsuo and Kitayama $\left.{ }^{37}\right)$. In the present case, $\bar{X} / \bar{L}$ is fixed as 0.03 , indicating large crystal lamellae, to achieve ultradrawing.

When neglecting the transition regions $t$ and $t$, the crystallinity $X c$ becomes $\bar{Y} / \bar{X}$. Judging from the lamellar structure, the disregard of the transition zones is out of the framework of crystalline polymer morphology because of the existence of a folding region. Hence, $\bar{Y} / \bar{X}$ is set to be $2 / 3$, which is lower than $X c$, because the crystallinity volumes of the dry gel films prepared in decalin and paraffin solutions were $76.5 \%$ and $73.2 \%$, respectively. Using Equations (A12) and (A13) in the Appendix, the values of $t$ and $t^{\prime}$ were determined to be 0.051 and $0.166 \mathrm{~nm}$, respectively, for decalin and 0.047 and $0.129 \mathrm{~nm}$, respectively, for paraffin.

The parameter $\sigma_{\alpha}$ in Equation (A18) in the Appendix is associated with the shape of the profile. The profile of the SAXS arcs becomes broader with decreasing $\sigma_{\alpha}$, as the preferential orientation of lamellar assemblies parallel to the film surface become pronounced in equation (A18), as shown in the Appendix.

As shown in Figures 10a and b, the parameter values that give the best fit between experimental and theoretical curves were similar. The parameters $\sigma_{y} / \bar{Y}$ and $\sigma_{z} / \bar{Z}$ for decalin were slightly lower than those for paraffin, indicating a somewhat narrow size distribution of lamellar thickness. Interestingly, the other parameters that gave the best fit between the experimental and theoretical results had similar values. This finding indicates that the crystal lamellar structure in the dry gel film prepared in decalin is essentially the same as that in the film prepared in paraffin, although the gelation mechanisms in the solvents are quite different.

To justify this result, theoretical patterns were calculated, and Figures 10e and $f$ show the results for decalin and paraffin. The same parameter values used for the calculation of the scattered intensity in Figures 10a and $\mathrm{b}$ are used. The calculated patterns are in good agreement with the observed patterns. The value of $\sigma_{\alpha}(=3)$ for decalin, which is lower than that for paraffin $(=5)$, reveals broad arcs, indicating that the orientation of the lamellar assemblies for decalin are broader than those for paraffin with respect to the film thickness direction.

The question arises as to whether the lamellae in the dry gel films prepared in decalin and paraffin have similar structures despite the different gelation mechanisms, which are shown in Figures 1-9. To address this question, models (Figures 11a-f) are proposed.

In accordance with the previous results for the decalin solvent, ${ }^{12}$ the optimum concentration to elongate up to the maximum draw ratio (400 times) was $0.65 \mathrm{~g}$ per $100 \mathrm{ml}$ for this UHMWPE. On the other hand, $1 \mathrm{~g}$ per $100 \mathrm{ml}$ was the best concentration to prepare UHMWPE/filler composites, as discussed elsewhere. ${ }^{38-42}$ In previous experiments, ${ }^{12}$ the successful drawing up to 400 times decreased as the concentration moved from $0.65 \mathrm{~g}$ per $100 \mathrm{ml}$. Thus, it may be expected that most of the chains in the regime of low concentration are random coils with coupling entanglements that are predominantly intermolecular in nature. On the other hand, solutions corresponding to the regime of high concentrations such as $1 \mathrm{~g}$ per $100 \mathrm{ml}$ are thought to consist of interpenetrating random coils, which form a large number of coupling entanglements that are intra- and intermolecular. A concentration of $1 \mathrm{~g}$ per $100 \mathrm{ml}$ corresponds to these conditions in Figure 11a. When quenching, the solution was divided into polymer-rich and polymer-poor phases by liquid--liquid phase separation, optically anisotropic elements appeared in the polymer-rich phase, and optically anisotropic rods could be constructed by the coagulation of anisotropic scattering elements associated with loosely folded structures. That is, crystallization occurred in the later stage, as shown in Figure 11b. By the progression of crystallization, decalin solvents were removed from the gel, and this facilitates the gel syneresis. This procedure promoted crystallization and formed crystal lamellae as shown in Figure 11c.

On the other hand, UHMWPE chains in paraffin solvent were crystallized at $106-108^{\circ} \mathrm{C}$, and the gelation time was $\sim 1200 \mathrm{~s}$ as shown in Figure 1d. This behavior is different from the behavior in decalin because the gelation of UHMWPE chains occurred at $83^{\circ} \mathrm{C}$ after $3250 \mathrm{~s}$. The paraffin solution viscosity is much lower than that of the decalin solution in spite of the rapid crystallization at higher temperatures. From the low viscosity in Figure 1a, it seems that the UHMWPE chains in paraffin forms comb-like structures locally by crosslinking neighboring chains as shown in Figure 11d. The low viscosity of UHMWPE in paraffin is quite different from that in UHMWPE-diphenyl and diphenylether co-solvents. As for these cosolvents, ${ }^{49}$ the sudden shrinkage of the gel occurred at temperature $147^{\circ} \mathrm{C}$, and UHMWPW became solid in the solvent, indicating no formation of a suitable level of entanglements per molecules because of poor solvent relative to PE. Thus, the overlapping points in Figure 11d play a role analogous to chemical crosslinking points in the formation of swollen gels. 

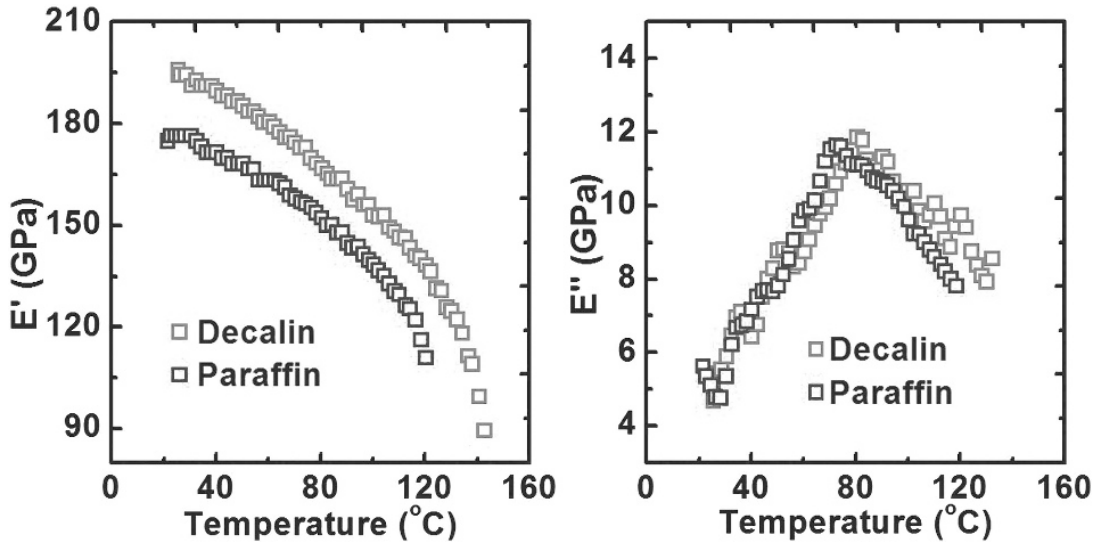

Figure 12 Temperature dependence of (a) the storage modulus and (b) the loss modulus of the ultradrawn dry gel films prepared in decalin and paraffin. A full color version of this figure is available at the Polymer Journal online.

In spite of the rapid crystallization at high temperatures, the gel syneresis was extremely slow. Actually, most of the paraffin solvent was contained in the gel and did not flow out from gel. Certainly, the liquid-liquid phase separation was advanced by chain diffusion as shown in Figure 11e, but the gelation/crystallization speed was extremely slow in comparison with UHMWPE chains in decalin. This is probably because of the good affinity between paraffin and PE because these substances have similar alkyl main chains. In Figure 11e, gels prepared in paraffin are almost in an equilibrium state. Because the gel syneresis in paraffin was extremely small, drying was done forcibly by blotting solvent from gel surfaces using filter paper as discussed above. By changing filter paper, the paraffin solvent in the gels was blotted gradually. Then, paraffin residues were removed in hexane. During the removal process, the UHMWPE chains formed large folded lamellae. In this case, crystal lamellae like those in Figure 11f can formed. The dry gel films prepared in paraffin composed of lamellae were similar to those in dry gel film prepared in decalin.

These dry gel films prepared in decalin and paraffin could be elongated up to 200 and 150 times, respectively, in a hot oven at 135 $140^{\circ} \mathrm{C}$. Figures $12 \mathrm{a}$ and $\mathrm{b}$ show the storage and loss moduli for both films. The storage modulus for decalin reached ca. $195 \mathrm{GPa}$. This value was almost equal to the values obtained elsewhere. ${ }^{10,12}$ On the other hand, the storage modulus for paraffin was $180 \mathrm{GPa}$. The elongation mechanisms for the dry gel films prepared in decalin and paraffin were the same, leading to crystal transformation from folded to extended chains, although the gel structure and gelation mechanism were quite different. The loss moduli for both films had $\alpha$ dispersion around $80^{\circ} \mathrm{C}$ associated with crystal dispesion. ${ }^{15}$

Incidentally, SAXS pattern for the ultradrawn films showed sharp streaks in the horizontal direction, indicating scattering from voids parallel to the oriented fibrils. These SAXS patterns suggest no existence of so-called 'kebab' structures observed in commercially spun fibers. This supports a high storage modulus beyond $180 \mathrm{GPa}$ near the crystal lattice modulus along crystal chain axes. ${ }^{10}$ Actually, the moduli of commercial fibers prepared by gel spinning are less than ca. $130 \mathrm{GPa}$.

\section{CONCLUSIONS}

Gelation mechanisms of UHMWPE solutions prepared in decalin and paraffin were investigated because both solvent are used for gel spinning in commercial production. Solutions were prepared by heating well-blended polymer/solvent mixtures at $145^{\circ} \mathrm{C}$ for $30 \mathrm{~min}$.
The gelation mechanism was investigated using light scattering to observe phase separation due to concentration fluctuations. The logarithm of scattered light intensity $\ell n(I)$ increased linearly with time during the initial stage of liquid-liquid phase separation when the solutions had been quenched to a desired temperature. Such behaviors occurred in the range $65-87^{\circ} \mathrm{C}$ for decalin and in the range $95-111^{\circ} \mathrm{C}$ for paraffin. This phenomenon could be analyzed within the framework of the linear theory of spinodal decomposition proposed by $\mathrm{Cahn}^{43}$ in terms of the phase separation of solution. In such super-cooled solutions, for decalin, any crystallites could not be detected by X-ray diffraction during the initial stage of phase separation, ensuring a linear increase in logarithm plots $\ell n(I)$, the scattered intensity, against time. This result suggests that the gelation of UHMWPE in decalin is generated in the polymer-rich phase (the heterogeneous network system) during phase separation, and then the phase separation plays a driving force to cause gelation/crystallization. As for paraffin solvent, the initial-phase separation was similar to that for decalin in the temperature range $95-105^{\circ} \mathrm{C}$. The very smaller X-ray peak in the time period $0-400 \mathrm{~s}$ was due to rapid crystallization after the initial high-temperature stage. However, no gel syneresis occurred in paraffin, even by quenching to room temperature, even after 1 month. Hv scattering showed no pattern or an extremely indistinct circular type pattern, and the corresponding POM images showed indistinct continuous structures when quenched to room temperature, indicating no growth of original crystal nuclei. This tendency was maintained, independent of time. In contrast, the solution prepared in decalin showed no Hv pattern during the initial stage, ensuring that there was a linear increase in $\ell n(I)$ against time in the temperature range $65-75^{\circ} \mathrm{C}$, but the pattern became clearer with time and developed an X-type pattern, and the corresponding POM images became continuous network structures. Gel syneresis played an important role to remove decalin from the gels and to promote the growth of large crystal lamellae. Gel syneresis in paraffin was extremely small, and the paraffin was blotted with filter paper placed on the gel surface. The residual paraffin was removed in hexane. The fitting between experimental and theoretical results for SAXS intensity distributions in the vertical direction as well as the SAXS patterns indicated that the lamellae in the dry gel films prepared in the two solvents had similar morphologies. The films were elongated more than 150 times by the transformation from folded to extended crystal chains. The storage modulus at $\sim 20^{\circ} \mathrm{C}$ was over $180 \mathrm{GPa}$ for both films. 


\section{ACKNOWLEDGEMENTS}

This work was supported by Natural Science Foundation of China (no. 21074016)

1 Smith, P. \& Lemstra, P. Ultra-high-strength polyethylene filaments by solution spinning/drawing. J. Mater. Sci. 15, 505-514 (1980).

2 Smith, P., Lemstra, P. J., Pijpers, J. P. L. \& Kiel, A. M. Ultra-drawing of high molecular weight polyethylene cast from solution. Colloid Polym. Sci. 259, 1070-1080 (1981).

3 Kanamoto, T., Tsuruta, A., Tanaka, K., Takeda, M. \& Porter, R. S. On ultra-high tensile modulus by drawing single crystal mats of high molecular weight Polyethylene. Pol. J. 15, 327-329 (1983)

4 Kanamoto, T., Tsuruta, A., Tanaka, K., Takeda, M. \& Porter, R. S. Super-drawing of ultrahigh molecular weight polyethylene. 1. Effect of techniques on drawing of single crystal mats. Macromolecules 21, 470-477 (1988).

5 Syouji, M., Sawai, D., Kanamoto, T. \& Ohama, T. Melt-drawing of ultrahigh molecular weight polyethylene: effect of molecular weight. Fiber 60, 316-321 (2004).

6 Nakae, M., Uehara, H., Kanamoto, T., Ohama, T. \& Porter, R. S. Melt drawing of ultrahigh molecular weight polyethylene: comparison of Ziegler- and metallocene-catalyzed reactor powders. J. Polym. Sci. B Polym. Phys. 37, 1921-1930 (1999).

7 Matsuo, M. \& Manley, R. S. J. Ultradrawing at room temperature of high molecular weight polyethylene. Macromolecules 15, 985-987 (1982).

8 Matsuo, M. K. I. \& Abumiy, N. Deformation mechanism of dry gel polyethylene up to a draw ratio of 300. Sen-i-Gakkaishi 40, 275-284 (1984).

9 Matsuo, M., Sawatari, C., lida, M. \& Yoneda, M. Ultradrawing of high molecular weight polyethylene films Produced by gelation/crystallization from solution: effect of the number of entanglements. Pol. J. 17, 1197-1208 (1985).

10 Matsuo, M. \& Sawatari, C. Elastic modulus of polyethylene in the crystal chain direction as measured by x-ray diffraction. Macromolecules 19, 2036-2040 (1986).

11 Matsuo, M. \& Sawatari, C. Temperature dependence of the crystal lattice modulus and the Young's modulus of polyethylene. Macromolecules 21, 1653-1658 (1988)

12 Ogita, T., Yamamoto, R., Suzuki, N., Ozaki, F. \& Matsuo, M. Molecular-weight dependence of morphology and mechanical properties of ultrahigh-molecular-weight polyethylene gel films. Polymer 32, 822-834 (1991).

13 Ogita, T., Kawahara, Y., Nakamura, R., Ochi, T., Minagawa, M. \& Matsuo, M. Drawability of ultrahigh molecular weight polyethylene single-crystal mats. Macromolecules 26, 4646-4651 (1993).

14 Furuhata, K., Yokokawa, T. \& Miyasaka, K. Drawing of ultrahigh-molecular-weight polyethylene single-crystal mats. J. Polym. Sci. Polym. Phys. Edit 22, 133-138 (1984).

15 Matsuo, M. Sawatari, C. \& Ohhata, T. Dynamic mechanical studies on the crystal dispersion using ultradrawn polyethylene films. Macromoleculars 21, 1317-1324 (1988).

16 Ogita, T., Suzuki, N., Ozaki, F., Sawatari, C. \& Matsuo, M. Morphology and mechanical properties of polyethylene gel films prepared from decalin and p-xylene solutions. Seni-Gakkaishi 46, 481-486 (1990).

$17 \mathrm{Hu}$, P. P., You, X. L. \& Liu, Z. F. A process for producing fiber of ultra high molecular weight polyethylene European patent 07816520.6 (2009)

18 Kobayashi, M., Nakaoki, T. \& Ishihara, N. Molecular conformation in glasses and gels of syndiotactic and isotactic polystyrenes. Macromolecules 23, 78-83 (1990).

19 Kobayashi, M. \& Kozasa, T. Conformational ordering process on physical gelation of syndiotactic polystyrene/solvent systems revealed by time-resolved infrared spectroscopy. Appl. Spectrosc 47, 1417-1424 (1993).

20 Tan, H., Moet, A., Hiltner, A. \& Baer, E. Thermoreversible gelation of atactic polystyrene solutions. Macromolecules 16, 28-34 (1983).

21 Gan, J. Y. S., Francois, J. \& Guenet, J. M. Enhanced low-angle scattering from moderately concentrated solutions of atactic polystyrene and its relation to physical gelation. Macromolecules 19, 173-178 (1986)

22 Klein, M. \& Guenet, J. M. Effect of polymer chain microstructure on solvent crystallization: implications on polymer solvation and on physical gelation. Macromolecules 22, 3716-3725 (1989).

23 Klein, M., Brulet, A. \& Guenet, J. M. Molecular structures in isotactic polystyrene thermoreversible gels. Macromolecules 23, 540-548 (1990).

24 Guenet, J. M. On the molecular structure of isotactic polystyrene physical gels as revealed by DSC and neutron diffraction. Macromolecules 19, 1961-1968 (1986).

25 Boyer, R. F., Baer, E. \& Hiltner, A. Concerning gelation effects in atactic polystyrene solutions. Macromolecules 18, 427-434 (1985).

26 Izumi, Y., Suzuki, J., Katano, S. \& Funahashi, S. Gelation mechanism of atactic polystyrene/carbon disulfide as revealed by neutron diffraction. Physica B 213-214, 724-726 (1995).

\section{APPENDIX}

Figure $10 \mathrm{~g}$ shows coordinates used to calculate the scattered intensity from an oriented lamellar assembly in the two-dimensional plane. In this model system, the $\mathrm{X}_{3}$ axis corresponds to the film normal direction and the $\mathrm{X}_{1}$ axis is parallel to the direction of an incident beam with unit vector defined as $\boldsymbol{s}_{\boldsymbol{o}}$. The intensity distribution is
27 Kanaya, T., Ohkura, M., Kaji, K., Furusaka, M. \& Misawa, M. Structure of poly(vinyl alcohol) gels studied by wide- and small-angle neutron scattering. Macromolecules 27, 5609-5615 (1994).

28 Matsuo, M., Kawase, M., Sugiura, Y., Takematsu, S. \& Hara, C. Phase separation behavior of poly(vinyl alcohol) solutions in relation to the drawability of films prepared from the solutions. Macromolecules 26, 4461-4471 (1993).

29 Matsuo, M., Sugiura, Y., Takematsu, S., Ogita, T., Sakabe, T. \& Nakamura, R. Relationship between drawability of poly(vinyl alcohol) films prepared from semi-dilute solutions and phase separation of the solutions studied in terms of stereo-regularity and degree of polymerization. Polymer 38, 5953-5967 (1997).

30 Matsuo, M., Hashida, T., Tashiro, K. \& Agari, Y. Phase separation of ultrahigh molecular weight isotactic polypropylene solutions in the gelation process estimated in relation to the morphology and mechanical properties of the resultant dry gel films. Macromolecules 35, 3030-3040 (2002)

31 Matsuo, M., Tanaka, T. \& Ma, L. Gelation mechanism of agarose and $\kappa$-carrageenan solutions estimated in terms of concentration fluctuation. Polymer 43, 5299-5309 (2002).

32 Matsuo, M., Miyoshi, S., Azuma, M., Bin, Y. Z., Agari, Y., Sato, Y. \& Kondo, A. Phase separation of several kinds of polyethylene solution under the gelation/crystallization process. Macromolecules 38, 6688-6699 (2005).

33 Matsuo, M., Yamanaka, A. \& Nakano, Y. Gelation mechanism of ultra-high-molecularweight polyethylene (UHMWPE) chains in dispersion solutions containing multiwall carbon nanotubes (MWNTs) analyzed in terms of liquid-liquid phase separation. Pure Appl. Chem. 81, 513-524 (2009).

34 Luo, Y. L., Kayakabe, S., Xi, Y., Bin, Y. Z., Xu, C. Y. \& Matsuo, M. Characteristics of several kinds of polyethylene gel estimated by small-angle light scattering under cross polarization. J. Polym. Sci. B Polym. Phys. 49, 384-397 (2011).

35 Matsuo, M., Miyoshi, S., Azuma, M., Nakano, Y. \& Bin, Y. Z. Polarized small-angle light scattering from gels estimated in terms of a statistical approach. Phys. Rev. E 72, 041403-041410 (2005).

36 Matsuo, M., Sawatari, C., Tsuji, M. \& Manley, R. S. J. One-dimensional mathematical treatment of small-angle X-ray scattering from a system of alternating lamellar phases. J. Chem. Soc., Perkin Trans. 2 79, 1593-1605 (1983).

37 Matsuo, M. \& Kitayama, C. Two-dimensional mathematical treatment of small-angle X-ray scattering from a system of alternating lamellar phases with orientation distribution. Pol. J. 17, 479-497 (1985).

$38 \mathrm{Xu}$, C. Y., Agari, Y. \& Matsuo, M. Mechanical and electric properties of ultra-highmolecular weight polyethylene and carbon black particle blends. Polym. J. 30, 372-380 (1998).

39 Bin, Y. Z., Xu, C. Y., Zhu, D. \& Matsuo, M. Electrical properties of polyethylene and carbon black particle blends prepared by gelation/crystallization from solution. Carbon 40, 195-199 (2002).

40 Xi, Y., Ishikawa, H., Bin, Y. Z. \& Matsuo, M. Positive temperature coefficient effect of LMWPE-UHMWPE blends filled with short carbon fibers. Carbon 42, 1699-1706 (2004).

41 Bin, Y. Z., Kitanaka, M., Zhu, D. \& Matsuo, M. Development of highly oriented polyethylene filled with aligned carbon nanotubes by gelation/crystallization from solutions. Macromolecules 36, 6213-6219 (2003).

42 Shi, X. M., Bin, Y. Z., Hou, D. S. \& Matsuo, M. Surface characterization for ultrahigh molecular weight polyethylene/hydroxyapatite gradient composites prepared by the gelation/crystallization method. ACS Appl. Mater. Interfaces 5, 1768-1780 (2013).

43 Cahn, J. W. Phase separation by spinodal decomposition in isotropic systems. J. Chem. Phys. 42, 93-99 (1965).

44 Cahn, J. W. \& Hilliard, J. E. Free energy of a nonuniform system. I. Interfacial free energy. J. Chem. Phys. 29, 258-267 (1958).

45 Van Aartsen, J. J. Theoretical observations on spinodal decomposition of polymer solutions. Eur. Polym. J. 6, 919-924 (1970)

46 Stein, R. S. \& Wilson, P. R. Scattering of light by polymer films possessing correlated orientation fluctuations. J. Appl. Phys. 33, 1914-1922 (1962).

47 Rhodes, M. B. \& Stein, R. S. Scattering of light from assemblies of oriented rods. J. Polym. Sci. A2 Polym. Phys. 7, 1539-1558 (1969).

48 Matsuo, M., Kakei, K., Nagaoka, Y., Ozaki, F., Murai, M. \& Ogita, T. A light scattering study of orientation of liquid-crystalline rodlike textures of poly(gamma-benzyl-Lglutamate) in an electric field by saturation electric birefringence method. J. Chem. Phys. 75, 5911-5924 (1981).

49 Ogita, T., Ochi, T., Li, Y. B. \& Matsuo, M. Morphology and mechanical properties of ultrahigh-molecular-weight polyethylene films prepared from solutions in mixtures of diphenyl and diphenylether as co-solvent. Colloid. Polym. Sci 272, 536-541 (1994).

50 Blundell, D. One-dimensional models for small-angle X-ray diffraction from crystalline polymers. I. General model. Acta Cryst. A 26, 472-476 (1970).

observed as a function of the scattering angle $2 \theta$ and the azimuthal angle $\mu$, and $\boldsymbol{s}^{\prime}$ is a unit vector along the scattered X-ray beam. To simplify the calculations, it is assumed that the crystalline lamellae are rectangular in cross-section and situated in the $0-\mathrm{X}_{2} \mathrm{X}_{3}$ plane. The center of gravity of each crystalline lamella is in the direction of the $u$ axis. 
The dimensions of the $j$-th lamellae are represented by $\bar{L}$ and $\bar{Y}_{j}$. The $\mathrm{u}$ axis is fixed on the $0-X_{2} X_{3}$ plane. The size of lamella in the $X_{1}$ direction is neglected, because it affects only the magnitude of the scattered intensity but is independent of the intensity distribution. In this model system, it is assumed that all the lamellae have same length $\bar{L}$. The distance between the $i$-th and the $(j+1)$-th lamellae is defined as $Z_{j}$, and $\alpha$ denotes the orientational angle between $X_{3}$ and the $u$ axes. To represent the variation of the density along the $u$ axis, two schematic diagrams are proposed. Incidentally, for mathematical simplicity, the variation along the $v$ axis, which is parallel in the direction of lamellar length, is neglected.

Figures $10 \mathrm{~h}$ and i show schematic diagrams that represent that the density variations of the two phases, specified by positive and negative deviations from the average density of the system, in which Figure $10 \mathrm{~h}$ is specified by an arbitrary function, and Figure 10i is a trapezoidal function. The lengths $Y_{j}$, and $Z_{j}$, are those of the representative phases, and $X_{j}$ is the total length (the repeat distance) of the $j$-th pair. The lengths $t$ and $t^{\prime}$ of the transition zones are assumed to be constant, whereas the lengths $Y_{j}$, and $Z_{j}$ are assumed to fluctuate independently from their average values, $\bar{Y}$ and $\bar{Z}$.

Considering the geometrical arrangement described in Figures $10 \mathrm{~g}-\mathrm{i}$, the scattering amplitude from the $N$ pairs of phase is given by

$$
\begin{aligned}
a(s) & =-L / 2 \int_{-L / 2}^{L / 2} \int_{u_{1}}^{u_{N+1}} \rho(u) \exp [-2 \pi i(b u-a v)] d u d v \\
& =\sum_{j=1}^{N} \int_{-L / 2}^{L / 2} \int_{u_{j}}^{u_{j+1}} \rho(u) \exp [-2 \pi i(b u-a v)] d u d v
\end{aligned}
$$

where $a$ and $b$ in Equation (A1) are given as a function of $s(=\sin 2 \theta / \lambda)$ as follows:

$$
a=s \sin (\alpha-\mu)
$$

and

$$
b=s \cos (\alpha-\mu)
$$

where $N$ is the number of pairs of domains, $\rho(u)$ in Equation (A1) denotes the density distribution in the direction of $u$ axis and can be rewritten as $\xi_{1}(u)\left(0 \leqslant y_{j} \leqslant Y_{j}\right)$ and $\xi_{2}(u)\left(0 \leqslant z_{j} \leqslant Z_{j}\right)$. Then

$$
\begin{aligned}
& a(s)=\sum_{j=1}^{N} \int_{-L / 2}^{L / 2} \int 0^{Y_{j}} \xi_{1}\left(y_{j}\right) \exp \left[-2 \pi i\left\{b\left(u_{j}+y_{j}\right)-a v\right\}\right] d y_{j} d v \\
& +\sum_{j=1}^{N} \int_{-L / 2}^{L / 2} \int_{u_{j}}^{Z_{j}} \xi_{2}\left(z_{j}\right) \exp \left[-2 \pi i\left\{b\left(u_{j}+Y_{j}+z_{j}\right)-a v\right\}\right] d z_{j} d v \\
& =\sum_{j=1}^{N} \exp \left(-2 \pi i b u_{j}\right) f_{j}+\sum_{j=1}^{N} \exp \left\{-2 \pi i b\left(u_{j}+Y_{j}\right)\right\} g_{j}
\end{aligned}
$$

where

$$
\begin{aligned}
f_{j} & =\int_{-L / 2}^{L / 2} \int_{0}^{Y_{j}} \xi_{1}\left(y_{j}\right) \exp \left[-2 \pi i\left(b y_{j}-a v\right)\right] d y_{j} d v \\
& =\frac{\sin (\pi a \bar{L})}{\pi a} \int_{0}^{Y_{j}} \xi_{1}\left(y_{j}\right) \exp \left(-2 \pi i b y_{j}\right) d y_{j} \\
g_{j} & =\int_{-L / 2}^{L / 2} \int_{0}^{Z_{j}} \xi_{2}\left(z_{j}\right) \exp \left[-2 \pi i\left(b z_{j}-a v\right)\right] d z_{j} d v \\
& =\frac{\sin (\pi a \bar{L})}{\pi a} \int_{0}^{Z_{j}} \xi_{2}\left(z_{j}\right) \exp \left(-2 \pi i b z_{j}\right) d z_{j}
\end{aligned}
$$

Considering the orientation distribution function $p(\alpha)$ of the lamellar assembly with respect to the $\mathrm{X}_{3}$ axis, the average scattered intensity is given by

$I(s)=\int_{0}^{2 \pi} p(\alpha) a(s) a *(s) d \alpha / \int_{0}^{2 \pi} p(\alpha) d \alpha=\left(I_{1}+I_{2}+I_{3}+I_{4}\right) / \int_{0}^{2 \pi} p(\alpha) d \alpha$

where

$$
\begin{aligned}
& I_{1}=\sum_{j=1}^{N} \sum_{k=1}^{N} \int_{0}^{2 \pi} p(\alpha) \exp \left\{-2 \pi i b\left(u_{j}-u_{k}\right)\right\} f_{j} f_{k} * d \alpha \\
& I_{2}=\sum_{j=1}^{N} \sum_{k=1}^{N} \int_{0}^{2 \pi} p(\alpha) \exp \left\{-2 \pi i b\left(u_{j}+Y_{j}-u_{k}-Y_{k}\right)\right\} g_{j} g_{k} * d \alpha \\
& I_{3}=\sum_{j=1}^{N} \sum_{k=1}^{N} \int_{0}^{2 \pi} p(\alpha) \exp \left\{-2 \pi i b\left(u_{j}-u_{k}-Y_{k}\right)\right\} f_{j} g_{k} * d \alpha \\
& I_{4}=\sum_{j=1}^{N} \sum_{k=1}^{N} \int_{0}^{2 \pi} p(\alpha) \exp \left\{-2 \pi i b\left(u_{j}+Y_{k}-u_{k}\right)\right\} g_{j} f_{k} * d \alpha
\end{aligned}
$$

where $a(s), f^{\star}$ and $g^{\star}$ denote complex conjugates of $a(s), f$ and $g$, respectively. The scattered intensity corresponds to the real parts of Equations (A7-1) to (A7-4) and the real part of $I_{3}$ is equivalent to that of $I_{4}$. Incidentally, the sum of the each imaginary part of Equations (A7-1) to (A7-4) is zero, if the lamellae possess a center of symmetry.

Following Blundell, ${ }^{50}$ the scattered intensity may be normalized as $a(s) a^{\star}(s) / N \bar{X} \bar{L}$, where $\bar{X}=(\bar{Y}+\bar{Z})$ denotes the mean periodic distance and therefore $N \bar{X} \bar{L}$ corresponds to the area of the assembly of lamellae. Thus, the total scattered intensity per unit area of scattering structure will be given by

$$
\frac{I}{N \bar{X} \bar{L}}=\frac{\left(I_{1}+I_{2}+2 I_{3}\right)}{N \bar{L} \bar{X} \int_{0}^{2 \pi} p(\alpha) d \alpha}=I_{B}-I_{C}
$$

Equation (A8) was given as a very complicated equation elsewhere. $^{37}$

From Figure 10i, the density distribution $\xi_{1}\left(y_{i}\right)$ of the $j$-th lamella (domain) with the thickness $Y_{j}$ denoting the positive density deviation from the average density of the system, is given by

$$
\begin{array}{rlrl}
\xi_{1}\left(y_{j}\right)= & \frac{\left(\rho_{c}-\bar{\rho}\right)}{t} y_{j} & 0 \leq y_{j} \leq t \\
\rho_{c}-\bar{\rho} & t_{j} \leq Y_{j}-t \\
& \left(\rho_{c}-\bar{\rho}\right)\left(Y_{j}-y_{j}\right) / t & Y_{j}-t_{j} \leq Y_{j}
\end{array}
$$

where $\rho_{c}$ is the electron density of the lamella (domain) with the thickness $Y_{j}$ and $\bar{\rho}$ is the average electron density of the whole sample. Similarly, the density distribution $\xi_{2}\left(z_{j}\right)$ of the medium with the length $Z_{j}$ surrounded by the $j$-th and $(j+1)$-th lamellae denotes the negative deviation, which is given by

$$
\begin{array}{rlrl}
\xi_{2}\left(z_{j}\right)= & \frac{\left(\rho_{a}-\bar{\rho}\right)}{t^{\prime}} z_{j} & & \leq y_{j} \leq t \\
& \rho_{a}-\bar{\rho} & t_{j}^{\prime} \leq Z_{j}-t^{\prime} \\
& \left(\rho_{a}-\bar{\rho}\right)\left(Z_{j}-z_{j}\right) / t^{\prime} & & Z_{j}-t_{j}^{\prime} \leq Z_{j}
\end{array}
$$

where $\rho_{a}$ is the electron density of the lamella (domain) with the length $Z_{j}$. In the present case, $\rho_{c}$ and $\rho_{a}$ correspond to the crystal and 
amorphous densities of PE, respectively. The average electron density $\bar{\rho}$ corresponding to the average density of specimen can be written by using volume crystallinity $X_{c}$ (or volume fraction) as follows:

$\bar{\rho}=X c \rho_{c}+(1-X c) \rho_{a}$

In addition, the transition zones $t$ and $t^{\prime}$ can be given by

$$
t=\frac{(1-X c)(p-X c)}{1-2 X c} \bar{X}
$$

and

$$
t^{\prime}=\frac{X c(p-X c)}{1-2 X c} \bar{X}
$$

where $p$ is given by $\bar{Y} / \bar{X}$.

Here it may be assumed that the variations of the lengths $Y_{j}$ and $Z_{j}$ are independent and are given by symmetrical Gaussian functions with the respective mean thickness of $\bar{Y}$ and $\bar{Z}$, and the standard deviations $\sigma_{y}$ and $\sigma_{z}$; thus,

$$
\begin{aligned}
& H\left(Y_{j}\right)=(2 \pi)^{-1 / 2} \sigma_{y}^{-1} \exp \left\{-\left(Y_{j}-\bar{Y}\right)^{2} / 2 \sigma_{y}^{2}\right\} \\
& h\left(Z_{j}\right)=(2 \pi)^{-1 / 2} \sigma_{z}^{-1} \exp \left\{-\left(Z_{j}-\bar{Z}\right)^{2} / 2 \sigma_{z}^{2}\right\}
\end{aligned}
$$

By using $\xi_{1}\left(y_{j}\right), \xi_{2}\left(z_{j}\right), H\left(Y_{j}\right), h\left(Z_{j}\right), \bar{\rho}, t$ and $t^{\prime}, I_{B}$ and $I_{C}$ can be represented as a function of $\sigma_{y} / \bar{Y}, \sigma_{z} / \bar{Z}$ and $\bar{X} / \bar{L}$, as described elsewhere. ${ }^{36,37}$ The number of scattering maxima increased and peak profile became sharper with decreasing $\sigma_{y} / \bar{Y}$ and $\sigma_{z} / \bar{Z}$. Considering a real system, the position of the specimens sampled by the X-ray beam contains a distribution of the number $N$ of pairs of the two phases. This concept must be introduced to smear out the many subsidiary maxima that appear at lower scattering angle. This has been taken into consideration for several cases by introducing a discrete symmetrical distribution of $N$ with standard deviation $\sigma_{N}=3$. This distribution is written as

$$
P(N)=\exp \left\{-\frac{(N-\bar{N})^{2}}{2 \sigma_{N}^{2}}\right\} / \sum_{N=1}^{2 \bar{N}-1} \exp \left\{-\frac{(N-\bar{N})^{2}}{2 \sigma_{N}^{2}}\right\}
$$

In Equation (A8), $I_{B}$ term is independent of $N$, whereas $I_{C}$ term contains $N$. Assuming the same type of distribution of $N$, the average values of the term $I_{C}$ is given by

$$
\left\langle I_{C}\right\rangle_{a v}=\sum_{N=1}^{2 \bar{N}-1} I_{C} P(N)
$$

In the present subsequent calculations, an average $\bar{N}$ is obtained by curve fitting in the present case (see Figure 10).

To complete this theoretical analysis, the distribution function of the lamellae with respect to the $\mathrm{X}_{3}$ axis must be defined. It is defined by

$$
p(\alpha)=\exp \left(-\sigma_{\alpha}^{2} \sin ^{2} \alpha\right)
$$

where $\sigma_{\alpha}$ is a parameter associated with the shape of $p(\alpha)$. When $\sigma_{\alpha}$ is large value, $p(\alpha)$ shows a sharp distribution. In contrast, when $\sigma_{\alpha}=0$, $p(\alpha)$ shows random distribution with maximum fluctuation. That is, the scattering arcs becomes narrower with increasing $\sigma_{\alpha}$. 